\title{
A Proof of the Bounded Graph Conjecture
}

\author{
Reinhard Diestel and Imre Leader
}

\begin{abstract}
An infinite graph is called bounded if for every labelling of its vertices with natural numbers there exists a sequence of natural numbers which eventually exceeds the labelling along any ray in the graph. We prove an old conjecture of Halin, which characterizes the bounded graphs in terms of four forbidden topological subgraphs.
\end{abstract}

\section{Introduction}

Let $\sigma, \tau: \mathbb{N} \rightarrow \mathbb{N}$ be two sequences of natural numbers. We say that $\sigma$ dominates $\tau$ if $\sigma(n) \geqslant \tau(n)$ for every $n$ greater than some $n_{0} \in \mathbb{N}$. Now let $G$ be an infinite graph, and assume the vertices of $G$ are labelled with natural numbers, say by $f: V(G) \rightarrow \mathbb{N}$. Then each ray (one-way infinite path) $R=x_{0} x_{1} \ldots$ in $G$ gives rise to a sequence $\tau_{R}:=\left(f\left(x_{0}\right), f\left(x_{1}\right), \ldots\right)$; we say that $\sigma$ dominates $R$ if $\sigma$ dominates this sequence $\tau_{R}$. We may then ask whether or not there exists a sequence $\sigma: \mathbb{N} \rightarrow \mathbb{N}$ which dominates all the rays in $G$ simultaneously. If this is the case, we say that the labelling $f$ of $G$ is bounded by $\sigma$; if not, $f$ is unbounded.

An unlabelled graph $G$ is said to be bounded if every labelling of its vertices is bounded. Thus, $G$ is bounded if and only if for every labelling $f: V(G) \rightarrow \mathbb{N}$ there exists a sequence $\sigma: \mathbb{N} \rightarrow \mathbb{N}$ which dominates every ray in $G$ with respect to $f$. If not, $G$ will be called unbounded.

Our main aim in this paper is to give a proof of what has become known as the 'bounded graph conjecture' [5]. This conjecture, proposed by Halin in 1964 (see [5]) but first published in [4], characterizes the bounded graphs by the exclusion of four simple prototypes of unbounded graphs as topological subgraphs. Not surprisingly, this characterization has some fundamental implications for the concept of boundedness.

Before we state the bounded graph conjecture in detail, let us look at a few examples of bounded and unbounded graphs. $K_{\omega}$, the complete graph on a countably infinite set of vertices, is clearly unbounded: choose any labelling that uses infinitely many distinct labels, and there will be rays whose labellings grow faster than any fixed $\mathbb{N} \rightarrow \mathbb{N}$ sequence.

Another trivial example of an unbounded graph is the following. For a cardinal $\kappa$, let $I_{\kappa}$ denote the disjoint union of $\kappa$ rays. Then $I_{\kappa}$ is unbounded for every $\kappa \geqslant 2^{\omega}$. Indeed, since there are only $2^{\omega}$ different $\mathbb{N} \rightarrow \mathbb{N}$ sequences, we can accommodate every possible sequence as the labelling of one of the rays of $I_{\kappa}$. The overall labelling of $I_{\kappa}$ then is clearly unbounded, and so $I_{\kappa}$ is unbounded. 
This trivial uncountable example of an unbounded graph is in fact the only uncountable aspect of the whole bounded graph problem: as we shall see, the task of classifying the bounded or unbounded graphs without uncountably many disjoint rays is an entirely 'countable' problem. (Indeed, we shall find that such a graph is bounded if and only if all its countable subgraphs are bounded (Corollary 7.3).)

Let us then look at some further countable examples. A graph with only countably many rays is bounded, due to the following well-known observation:

Lemma 1.1. Every countable family of sequences $\sigma_{n}: \mathbb{N} \rightarrow \mathbb{N}(n \in \mathbb{N})$ is dominated by a common sequence $\sigma: \mathbb{N} \rightarrow \mathbb{N}$.

Proof. For each $n \in \mathbb{N}$, set $\sigma(n):=\max \left\{\sigma_{0}(n), \ldots, \sigma_{n}(n)\right\}$.

For example, a single ray contains only countably many distinct rays as subgraphs (namely, all its 'tails'), and is therefore bounded.

A similar argument shows that, more generally, every locally finite connected graph $G$ is bounded. Indeed, given a labelling $f$ and any fixed vertex $v$ of $G$, it is easy to construct a sequence $\sigma_{v}$ which dominates all the rays in $G$ starting at $v$; then Lemma 1.1 provides a sequence which dominates all these (countably many) sequences $\sigma_{v}$, and hence bounds $f$.

The example of $K_{\omega}$ may suggest that countable graphs have to be dense in order to be unbounded. However, this is not the case. $T_{\omega}$, the tree of countably infinite regular degree, ${ }^{*}$ is also unbounded. To show this, we have to find a labelling $f: V\left(T_{\omega}\right) \rightarrow \mathbb{N}$ such that no $\mathbb{N} \rightarrow \mathbb{N}$ sequence dominates all the rays in $T_{\omega}$ with respect to $f$. This is easily done. In fact, all we ask is that $f$ be injective, i.e. that all the vertices of $T_{\omega}$ be labelled differently. Given $\sigma: \mathbb{N} \rightarrow \mathbb{N}$, we then choose a ray in $T_{\omega}$ as follows. Let $x_{0}$ be any vertex of $T_{\omega}$ whose label is greater than $\sigma(0)$. Since $f$ is injective, $x_{0}$ has a neighbour $x_{1}$ whose label exceeds $\sigma(1)$. Continuing in this manner, we find a ray $x_{0} x_{1} \ldots \subset T_{\omega}$ such that $f\left(x_{n}\right)>\sigma(n)$ for every $n$, so this ray is certainly not dominated by $\sigma$.

The following example of an unbounded graph is based on the same idea. Let $B$ be the graph obtained from a ray $v_{0} v_{1} \ldots$ by adding, for each $n \in \mathbb{N}$, a countably infinite set of independent $v_{3 n+1}-v_{3 n+3}$ paths of length 2 (Fig. 1). Any subdivision of $B$ will be called a bundle graph. (See $\S 2$ for the definition of subdivision etc.)

To show that $B$ is unbounded, let $f$ be any injective labelling of $B$, and assume we are given a sequence $\sigma: \mathbb{N} \rightarrow \mathbb{N}$. Let us specify a ray $R=x_{0} x_{1} \ldots$ as follows. Put $x_{0}:=v_{0}$ and $x_{1}:=v_{1}$. For $x_{2}$, we have an infinite choice of neighbours of $v_{1}$, all labelled differently. We may thus choose as $x_{2}$ a neighbour of $v_{1}$ whose label is greater than $\sigma(2)$. We continue with $x_{3}:=v_{3}$ and $x_{4}:=v_{4}$, where again we have an infinite choice for $x_{5}$. Proceeding in this manner, we

* This means that every vertex of $T_{\omega}$ has exactly $\omega$ neighbours; see $\S 2$. 


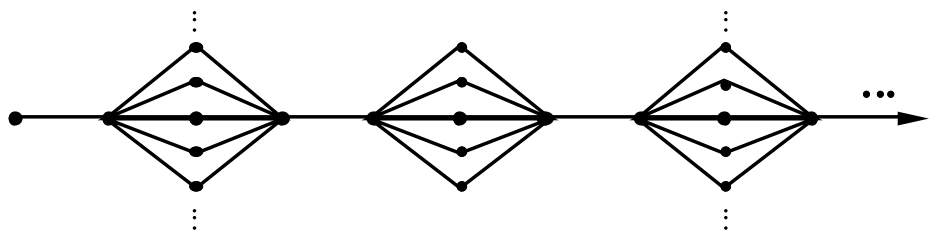

Figure 1. The prototype bundle graph $B$

may choose $R$ in such a way that $f\left(x_{n}\right)$ exceeds $\sigma(n)$ for every third value of $n$, and thus $\sigma$ fails to dominate $R$.

Our last example is similar to $B$. Let $F$ be the graph obtained from a ray $V=v_{0} v_{1} \ldots$ by adding disjoint rays $P_{2}, P_{4}, P_{6}, \ldots$ with $P_{k} \cap V=\left\{v_{k}\right\}$, and joining $v_{2 n+1}$ to all the new vertices of $P_{2 n+2}$ for every $n \in \mathbb{N}$ (Fig. 2). Any subdivision of $F$ will be called a fan graph.

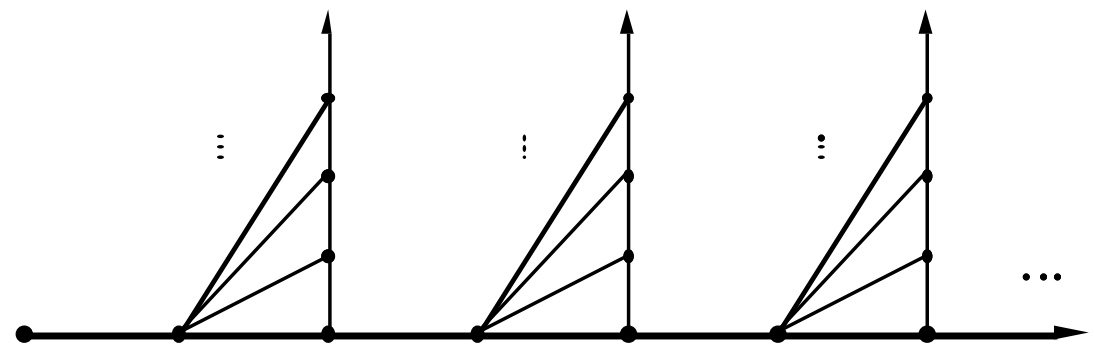

Figure 2. The prototype fan graph $F$

$F$ is again unbounded, the proof being essentially the same as for $B$. Indeed, given any injective labelling $f: V(F) \rightarrow \mathbb{N}$ and any sequence $\sigma: \mathbb{N} \rightarrow \mathbb{N}$, we can easily find a ray $R$ through $F$ whose labels exceed the corresponding values of $\sigma$ again and again. All we have to ensure when defining $R$ is that we start at $v_{0}$, and never use an edge of one of the paths $P_{k}$ in its 'upward' direction. (This would force us to trace out the entire tail of $P_{k}$, leaving us unable to return to a vertex of type $v_{2 n+1}$ with an infinite choice ahead.)

It is perhaps remarkable that $F$ can be made bounded by what would seem to be an inessential change. If the 'fans' in $F$ are flipped horizontally, i.e. if the vertices $v_{2 n+1}$ are joined to all the vertices of $P_{2 n}$ rather than to those of $P_{2 n+2}$ (add a ray $P_{0}$ ), the resulting graph is bounded. (This example is due to Halin; he found it so discouraging that it prompted him to doubt the possibility of a proof of his conjecture by elementary methods [5].)

We are now ready to state Halin's conjecture. If a graph $G$ has a subgraph isomorphic to a subdivision of a graph $X$, we say that $X$ is a topological 
subgraph of $G$.

Bounded Graph Conjecture. (Halin)

Assume the Continuum Hypothesis. A graph is bounded if and only if it contains none of the following graphs as a topological subgraph: $I_{\omega_{1}} ; T_{\omega} ; B ; F$.

The forward implication of the conjecture should be obvious: we have seen that the graphs $I_{2^{\omega}}, T_{\omega}, B$ and $F$ are all unbounded; these proofs remain essentially the same for subdivisions of the latter three graphs (and identical for $I_{\omega_{1}}=I_{2} \omega$ ), and it is clear that no bounded graph can have an unbounded subgraph.

A word concerning the Continuum Hypothesis $(\mathrm{CH})$. When Halin originally proposed the bounded graph conjecture (in connection with Rado's seminal paper on Universal graphs and universal functions [9]), it was understood to refer to countable graphs only, and $T_{\omega}, B$ and $F$ were the only forbidden (topological) subgraphs. There was no mention of $\mathrm{CH}$. Halin later strengthened the conjecture to include graphs of any cardinality, which resulted in the additional exclusion of $I_{2^{\omega}}$. This in turn suggested the assumption of $\mathrm{CH}$, as it seemed unclear whether or not $I_{\kappa}$ is bounded when $\kappa$ is a cardinal strictly between $\omega$ and $2^{\omega}$.

In fact, it is well-known among set theorists that this latter question is independent of the usual axioms of set theory. Martin's Axiom, for example, implies that every $I_{\kappa}$ with $\kappa<2^{\omega}$ is bounded, but it is consistent with ZFC that there exist $\kappa<2^{\omega}$ for which $I_{\kappa}$ is unbounded.

It should be stressed that our proof of the bounded graph conjecture is entirely free from such set theoretic considerations. To make this possible, we take the following (standard) approach. We shall define a cardinal $\mathfrak{b}$ in such a way that it is the least cardinal $\kappa$ for which $I_{\kappa}$ is unbounded (whatever the value of that cardinal may be), and then prove - without assuming $\mathrm{CH}$ - that a graph is bounded if and only if it contains none of $I_{\mathfrak{b}}, T_{\omega}, B$ or $F$ as a topological subgraph. Since $\omega<\mathfrak{b} \leqslant 2^{\omega}$, and hence $\omega_{1}=\mathfrak{b}=2^{\omega}$ with CH, this in particular implies the bounded graph conjecture as stated above.

It turns out that we need to show very little about $\mathfrak{b}$ to make this approach work. However, any reader who prefers to work with countable sets and is willing to accept $\mathrm{CH}$ will lose nothing by substituting 'countable' whenever he reads ' $<\mathfrak{b}$ '.

Before we get down to the proof of the bounded graph conjecture, let us look at some of its immediate implications. (See also §7.) As a first corollary, the conjecture implies that subdivisions of bounded graphs are again bounded, and that subdivisions of unbounded graphs are unbounded. The first of these two statements may not be unexpected: for countable graphs it follows without much effort from the definition of boundedness. The second assertion is more surprising. Indeed, it would seem that there should be graphs which 
are unbounded largely because they are dense (as our example of $K_{\omega}$ ), so that subdividing their edges sufficiently often might make them bounded.

Together with the obvious fact that subgraphs of bounded graphs are bounded, the assertion that subdivisions of unbounded graphs are again unbounded is equivalent to saying that boundedness is closed under taking topological subgraphs. We shall also derive a forbidden minor version of the bounded graph conjecture (Theorem 7.7), together with a corollary saying that boundedness is closed under taking minors (with finite branch sets).

Another consequence of the bounded graph conjecture is the fact that a graph is bounded if and only if all its countable subgraphs are boundedunless, of course, it contains uncountably many disjoint rays, in which case it is trivially unbounded (assuming $\mathrm{CH}$ ). Thus, boundedness is shown to be an essentially 'countable' property, irrespective of the cardinality of the graph considered.

Finally, the bounded graph conjecture implies that if a countable graph admits any unbounded labelling at all, then all its injective labellings are unbounded. (Recall that the latter is true for subdivisions of $T_{\omega}$, and for bundle and fan graphs.) It is therefore never difficult to find an unbounded labelling for an unbounded graph: its unboundedness lies properly in its structure, not in the complication of any particular labelling.

On the face of it, the bounded graph conjecture seems rather a bold conjecture to make. There appears to be nothing special about the four unbounded graphs which it claims are the only prototypes - indeed it would seem that playing with a few examples should reveal many more similar 'minimal' unbounded graphs. This may partly explain the fact that over the last 25 years there seem to have been few serious attempts at proving the conjecture.

Halin himself published his own partial results only very recently. In a paper written in 1986 [4], he proves the bounded graph conjecture for countable trees. Note that of the four forbidden graphs from the conjecture this involves only $T_{\omega}$; graphs of the other three types cannot even occur in a countable tree. A few years later, Halin extended this theorem to graphs whose blocks are rayless [5] (see also [2]). The extended result still involves only three of the four forbidden configurations, as fan graphs cannot occur. A very recent result of Halin, also found in [5], confirms the bounded graph conjecture for graphs not containing an infinite set of disjoint rays. (This last theorem builds on Halin's well-known structural characterization of these graphs by 'chain-like' decompositions.)

The following may help to motivate the bounded graph conjecture a little better, taking some of the arbitrariness out of its list of forbidden configurations. (It will also be used later, for a proof of Lemma 2.3.) Let $G$ be a connected unbounded graph. Since locally finite connected graphs are bounded, $G$ has a vertex $v$ of infinite degree. Let us assume that every edge incident with $v$ can be extended to a ray (which is not an enormous assumption), and 
let $R_{0}, R_{1}, \ldots$ be an infinite set of rays all starting at $v$. Can $R_{0}, R_{1}, \ldots$ be chosen pairwise independent (i.e., disjoint except for $v$ )? If so, they might be seen as the beginning of a subdivided $T_{\omega}$ in $G$, and we might try to find similar rays starting at other vertices of the $R_{n}$ to continue the construction.

If the rays $R_{0}, R_{1}, \ldots$ cannot be chosen independent, it seems reasonable to expect that we can choose them in such a way that there exists a finite set of vertices, and hence a single vertex $w$, which each of them meets. We may now apply König's theorem (see §2) to combine suitable initial segments of the rays $R_{n}$ into what might be the first 'bundle' of a bundle graph, or the first 'fan' of a fan graph in $G$.

Indeed, let us choose initial segments $P_{n}$ of the $R_{n}$ as follows. Let $P_{0}$ be the initial segment of $R_{0}$ that ends at $w$. Having defined $P_{0}, \ldots, P_{n}$ for some $n \in \mathbb{N}$, let $P_{n+1}$ be the initial segment of $R_{n+1}$ which ends at the first vertex of $R_{n+1}$ that is in $P_{0} \cup \ldots \cup P_{n}$. Let this vertex be called $w_{n+1}$, and let $k(n+1)$ be the minimal $k$ for which $w_{n+1} \in P_{k}$. Call $P_{k(n+1)}$ the predecessor of $P_{n+1}$.

Let $T$ be the tree whose vertices are the paths $P_{n}(n \in \mathbb{N})$, and in which each vertex $P_{n}$ (for $n=1,2, \ldots$ ) is joined by an edge to its predecessor $P_{k(n)}$. By König's theorem, $T$ contains a ray or has a vertex of infinite degree. If $T$ has a vertex of infinite degree, $P_{k}$ say, then $P_{k}$ contains a vertex $x$ such that $w_{n}=x$ for infinitely many $n>k$. The paths $P_{n}$ with $w_{n}=x$ are pairwise independent (i.e., disjoint except for $v$ and $x$ ), and they form a 'bundle' (Fig. 3).

On the other hand if $T$ contains a ray, without loss of generality starting at $P_{0}$, then the union of the paths $P_{n} \subset G$ which are the vertices of this ray are easily seen to form a 'fan' (Fig. 3). (Note that, by the construction of $T$, if $P_{m}$ succeeds $P_{n}$ on the ray in $T$ then $m>n$, so $P_{n}$ is the predecessor of $P_{m}$.)
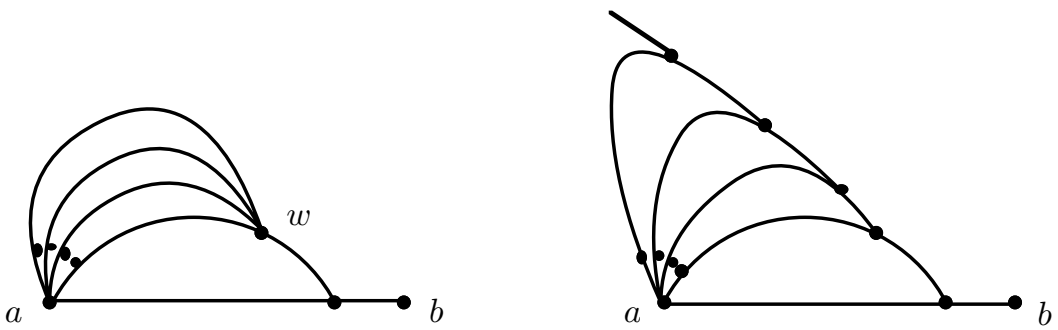

Figure 3. A bundle or a fan on a path $P_{k}=v \ldots w$

The main purpose of the above considerations is to persuade the reader that fans and bundles are indeed natural ingredients of unbounded graphs. It must be said, however, that these considerations have not taken us anywhere near a proof of the bounded graph conjecture. Indeed, one might well find various fans and bundles in an unbounded graph: the problem is that, in general, they will be far from disjoint. Our task will thus be to prove that unbounded graphs must contain fans and bundles in such abundance that it is possible to 
select an infinite set of them as tidily threaded on a ray as in the graphs $B$ and $F$.

The paper is organized as follows. We begin with a short section on notation and basic lemmas. This is followed by a section on normal spanning trees, the main structural tool in our proof, and another which contains two simple Ramsey-type lemmas concerning partitions of $T_{\omega}$. The bounded graph conjecture is then proved in Sections 5 and 6 , with a concluding section listing some immediate corollaries.

\section{Notation and basic lemmas}

All the graphs considered in this paper are undirected and simple, i.e. have neither loops nor multiple edges. The axiom of choice is assumed throughout the paper. The set of natural numbers, $\mathbb{N}$, includes 0 . The first infinite ordinal is denoted by $\omega$, the first uncountable ordinal by $\omega_{1}$.

Let $G$ be a graph. The edge set of $G$ will be denoted by $E(G)$, its vertex set by $V(G)$, and its number of vertices by $|G|$. When $H$ is a subgraph of $G$, denoted by $H \subset G$, we may simply say that $G$ contains $H$. If $x y$ is an edge (for $x, y \in V(G)$ ), then $y$ is a neighbour of $x$, and the two vertices are adjacent. The neighbourhood of a vertex is the set of its neighbours. $G$ is locally finite if the degree of each vertex, the number of its neighbours, is finite.

A path may be finite or infinite; its length is its number of edges. A path of length 0 is trivial. A one-way infinite path is a ray, and a graph is rayless if it contains no ray. Any infinite connected subgraph of a ray $R$ is a tail of $R$. We usually write a path as the sequence of its vertices, which gives these a natural order. It then makes sense to say that a path starts or ends at some particular vertex, that one vertex precedes another on it, and so on.

If $P=x_{1} \ldots x_{n}$ is a path, we write $\stackrel{\circ}{P}$ for the interior of $P$, the subpath $x_{2} \ldots x_{n-1}$. The vertices of $\stackrel{\circ}{P}$ are the inner vertices of $P$. Similarly, if $1 \leqslant i \leqslant j \leqslant n$, we set $P x_{i}:=x_{1} \ldots x_{i}, P \stackrel{\circ}{x}_{i}:=x_{1} \ldots x_{i-1}, x_{i} P x_{j}:=x_{i} \ldots x_{j}$, $x_{j} P:=x_{j} \ldots x_{n}$ and $\dot{x}_{j} P:=x_{j+1} \ldots x_{n}$ for subpaths of $P$. Analogous notation will be used for rays and for trees (so that $x T y$ is the unique path from $x$ to $y$ in the tree $T$ ), and for the concatenation of paths. For example, if $x$ is a common vertex of two paths $P$ and $Q$, then $P x Q$ denotes the 'walk' $P x \cup x Q$. The path $P x_{i}$ defined above is an initial segment of $P$, and $P$ an extension of $P x_{i}$. Note that a finite path is an initial segment of itself.

For $X, Y \subset G$, we call a finite path $P \subset G$ an $X-Y$ path if its endvertices are in $X$ and $Y$, respectively, and its inner vertices lie in $G \backslash(X \cup Y)$. When $X$ or $Y$ consists of only one vertex, we speak of (say) $x-Y$ or $X-y$ paths rather than $\{x\}-Y$ or $X-\{y\}$ paths.

If $X \subset V(G)$, we write $G[X]$ for the subgraph of $G$ induced by the vertices in $X$. For $H \subset G$ and $x \in G$, we write $H[x]$ for the subgraph of $H$ induced 
by those of its vertices $y$ for which $G$ contains an $x-y$ path that has no vertices in $H$ other than $y$. In particular, if $x$ is a vertex of $H$ then $H[x]$ is just the singleton $\{x\}$. On the other hand, if $x \notin H$ and $C$ is the component of $G \backslash H$ containing $x$, then $H[x]$ contains precisely those vertices of $H$ that have a neighbour in $C$.

Two or more paths are independent if their interiors are disjoint. If $A, B$ and $X$ are subgraphs of $G$ or subsets of $V(G)$, we say that $X$ separates $A$ from $B$ in $G$ if every $A-B$ path in $G$ contains a vertex of $X$. We use this notation even when $A$ and $B$ are not disjoint; of course, $X$ must then contain $A \cap B$.

When $T$ is a tree (an acyclic connected graph), we shall often pick a root $r \in V(T)$, and call $T$ rooted at $r$. This induces a natural partial order on $V(T)$, in which $x \leqslant y$ iff $x$ lies on the unique $r-y$ path $r T y$ in $T$. Informally, we think of this order as expressing the vertices' heights in $T$; we may then speak of $y$ being above $x$ if $y>x$, call it an upper neighbour of $x$ if it is above $x$ and adjacent to it, and so on.

Note that, for each vertex $z \in T$, the set of all vertices $x \leqslant z$ is linearly ordered; the subgraph it spans in $T$ will be denoted by $\lfloor z\rfloor$. The down-closure $\lfloor U\rfloor$ of a set $U \subset V(T)$ is the graph $\bigcup\{\lfloor u\rfloor \mid u \in U\} \subset T$. For any vertex $v$ of $T$, the subtree of $T$ spanned by all the vertices $x \geqslant v$ will be denoted by $T \geqslant v$. Similarly, we write $T^{>v}$ for $T^{\geqslant v} \backslash\{v\}$.

If $T \subset G$ and $V(T)=V(G)$, then $T$ is said to span the graph $G$. The order $\leqslant$ on $V(T)$ thus becomes an order on $V(G)$; if we wish to be more specific, we may denote it by $\leqslant_{T}$.

A subdivision of a graph $X$, denoted by $\mathrm{T} X$, is any graph obtained from $X$ by replacing its edges with independent paths of lengths $\geqslant 1$. The original vertices of $X$ are then called the branch vertices of the $\mathrm{T} X$; the other vertices of the $\mathrm{T} X$ are its subdividing vertices. $X$ is a topological subgraph of any graph containing a $\mathrm{T} X$. If $X$ is a tree rooted at some vertex $r$, then any $\mathrm{T} X$ will again be assumed to be rooted at $r$; the order on $V(X)$ is thus embedded in the tree order on the vertices of the $\mathrm{T} X$.

The following definition and lemma will not be needed until $\S 7$. They are included here for completeness, but the reader may wish to skip straight to König's theorem further below.

In analogy to our notation concerning topological subgraphs, we say that a graph $H$ is an $\mathrm{H} X$ (where ' $\mathrm{H}$ ' comes from 'homomorphism') if its vertex set admits a partition $\left\{V_{x} \mid x \in V(X)\right\}$ into subsets $V_{x}$ spanning connected subgraphs in $H$ so that $H$ contains a $V_{x}-V_{y}$ edge if and only if $x$ and $y$ are adjacent in $X$. The sets $V_{x}$ are called the branch sets of $H$. A graph $X^{\prime}$ is said to be a minor of $H$ if $H$ has a subgraph $H^{\prime}$ such that $H^{\prime}=\mathrm{H} X^{\prime}$ (or, equivalently, if $H=\mathrm{H} X$ with $\left.X^{\prime} \subset X\right)$.

Lemma 2.1. Let $X$ be a topological subgraph of a graph $G$. If $X$ is countable or locally finite, then $X$ is a minor of $G$ with all branch sets finite. 
Proof. By assumption, the components of $X$ all have countable vertex sets; choose an enumeration for each of them. Let $H$ be a TX contained in $G$. For each $x \in V(X)$, let $V_{x}$ be the subset of $V(H)$ that consists of $x$ and any subdividing vertices on $x-y$ edges of $X$ for which $y$ precedes $x$ in their common component of $X$. Note that each $V_{x}$ is finite, and that it spans a connected subgraph in $H$. Hence, $H$ is an $\mathrm{H} X$ as required.

The following theorem of König [7], a simpler version of his well-known Infinity Lemma, is a standard tool which we shall use frequently:

König's Theorem. Every infinite connected graph contains either a vertex of infinite degree or a ray.

We conclude with some less standard teminology.

It will often be useful to be able to combine several sequences of natural numbers into one fast growing sequence which dominates them all. For $\sigma: \mathbb{N} \rightarrow \mathbb{N}$, let $\sigma^{\prime}, \sigma^{\prime \prime}: \mathbb{N} \rightarrow \mathbb{N}$ be defined by setting $\sigma^{\prime}(n):=\max \{\sigma(0), \ldots, \sigma(n)\}$ and $\sigma^{\prime \prime}(n):=\sigma^{\prime}(2 n)$. Then $\sigma^{\prime \prime}$ dominates $\sigma^{\prime}$, which in turn dominates $\sigma$. The sequence $\sigma^{\prime \prime}$ will be called the majorant of $\sigma$. More generally, let $F$ be a family of $\mathbb{N} \rightarrow \mathbb{N}$ sequences dominated simultaneously by a sequence $\sigma$. We shall then also refer to the majorant $\sigma^{\prime \prime}$ of $\sigma$ as a majorant of the family $F$. Note that, by Lemma 1.1, every countable family of $\mathbb{N} \rightarrow \mathbb{N}$ sequences has a majorant.

The majorant of a single sequence is thus obtained by first making it monotonically increasing and then doubling its growth rate. It therefore 'catches up' with the original sequence even when shifted forward by any constant number of terms. This is the idea behind the following lemma, which is essentially due to Halin [5]:

Lemma 2.2. Let $F$ be a family of $\mathbb{N} \rightarrow \mathbb{N}$ sequences, and let $G$ be a graph with a labelling $V(G) \rightarrow \mathbb{N}$. If $R, R^{\prime} \subset G$ are rays sharing a tail, and if some $\sigma \in F$ dominates $R$, then any majorant of $F$ dominates $R^{\prime}$.

Let $a$ and $b$ be distinct vertices of a graph $G$. The union of an infinite set of independent $a-b$ paths of lengths $\geqslant 2$ will be called a bundle from $a$ to $b$, or an $a-b$ bundle. The interior of any of those paths is a fibre of the bundle; note that these fibres are pairwise disjoint. The interior of a bundle is the union of its fibres. An $a-b$ bundle $B$ is said to be on a path $P$ if $B \cap P=\{a, b\}, a$ precedes $b$ on $P$, and $a P b$ has length at least 2 . If $\left\{B_{1}, \ldots, B_{n}\right\}$ is a (possibly empty) set of disjoint bundles on a common path $P$, arranged in such a way that the first vertex of $P$ is in none of the $B_{i}$ and both vertices of $B_{i} \cap P$ precede both vertices of $B_{i+1} \cap P$ on $P$ for all $i<n$, then the union of $P$ and the $B_{i}$ is a partial bundle graph on $P$.

Similarly, the union of a ray $R$ with initial vertex $b$ and infinitely many paths from a vertex $a \notin R$ to $R \backslash\{b\}$ is called a fan from $a$ to $b$, or an $a-b$ 
$f a n$, if those paths are pairwise disjoint except for $a$ and have no inner vertices on $R$. The ray $R$ in such a fan is called its spine; the $a-R$ paths are its spokes. The interior of a fan $F$ from $a$ to $b$ is the graph $F \backslash\{a, b\}$. $F$ is said to be on a path $P$ if $F \cap P=\{a, b\}$ and $a$ precedes $b$ on $P$. If $\left\{F_{1}, \ldots, F_{n}\right\}$ is a (possibly empty) set of disjoint fans on a common path $P$, arranged in such a way that the first vertex of $P$ is in none of the $F_{i}$ and both vertices of $F_{i} \cap P$ precede both vertices of $F_{i+1} \cap P$ on $P$ for all $i<n$, then the union of $P$ and the $F_{i}$ is a partial fan graph on $P$.

Using these terms, we can now write down as a lemma a precise summary of the observations we made at the end of the Introduction. The proof of the lemma was sketched there; the details are left to the reader.

Lemma 2.3. Let $a$ and $b$ be distinct vertices of a graph $G$, and let $\mathcal{P}$ be an infinite set of $a-b$ paths in $G$ whose second vertices are pairwise distinct. The union $\bigcup \mathcal{P}$ of these paths then contains a bundle $B$ or a fan $F$. Moreover:

(i) The bundle $B$ can be chosen as an $a-x$ bundle on a path $P \in \mathcal{P}$ (where $x$ is some vertex on $P$ ), and so that every $a-x$ path in $B$ is an initial segment of a path in $\mathcal{P}$.

(ii) The fan $F$-with spine $R$, spokes $S_{0}, S_{1}, \ldots$, and $S_{n} \cap R=:\left\{v_{n}\right\}$ for each $n$-can be chosen so that $v_{n}$ precedes $v_{m}$ on $R$ whenever $n<m$, and each of the paths $S_{n} v_{n} R v_{n-1}$ is an initial segment of a path in $\mathcal{P}$ (where $v_{-1}$ is the starting vertex of $R$ ).

\section{Normal spanning trees}

In this section we introduce and develop the main structural tool for our proof, the concept of a normal spanning tree. A rooted spanning tree $T$ of a graph $G$ is called normal if the endvertices of every edge of $G$ are comparable in the tree order $\leqslant_{T}$ which $T$ induces on $V(G)$. Thus, if $r$ is the root of $T$ and $x y \in E(G)$, then either $x$ lies on the $r-y$ path in $T$ or $y$ lies on the $r-x$ path in $T$. Intuitively, all the edges of $G$ are 'along' branches of $T$, never 'across'.

It is easy to see that all finite connected graphs have normal spanning trees (obtained, for example, by a 'depth-first search'). Jung [6] characterized the infinite graphs having normal spanning trees; his characterization implies that every countable connected graph contains such a tree. Starting from Jung's result and using the theory of simplicial decompositions of graphs - see [1]Halin [3] was able to prove the following:

Theorem 3.1. (Halin)

If $G$ is connected and $G \nsupseteq \mathrm{T} K_{\omega}$, then $G$ has a normal spanning tree. 
When we come to prove that an unbounded graph $G$ must contain one of the four topological subgraphs listed in the bounded graph conjecture, we may thus assume that $G$ has a normal spanning tree: if not, it would contain a $\mathrm{T} K_{\omega}$, and hence a $\mathrm{T} T_{\omega}$, as desired.

We now collect together a number of simple facts about normal spanning trees to be used later. All these are easy to prove, and they should help the reader develop an intuition for the properties of a normal spanning tree.

Let $G$ be a fixed graph, and assume that $G$ contains a normal spanning tree $T$ with root $r$. Any reference to an order on the vertices of $G$ (such as 'above', 'below', down-closures $\lfloor x\rfloor$ and so on) will be assumed to refer to the order $\leqslant_{T}$ induced by $T$. A ray in $G$ will be called a normal ray if it starts at $r$ and is contained in $T$. The union of all normal rays, clearly a subtree of $T$, will be denoted by $T^{\prime}$.

Our first lemma translates the local defining property for $T$ (that every edge of $G$ runs vertically along $T$ ) into a more global separation property of $G$ :

Lemma 3.2. Let $x, y \in V(G)$. Then $\lfloor x\rfloor \cap\lfloor y\rfloor=\lfloor\inf \{x, y\}\rfloor$ separates $x$ from $y$ in $G$.

Proof. Let $P=x_{1} \ldots x_{n}$ be an $x-y$ path in $G$, and put $z:=\inf \{x, y\}$; we have to show that $P$ meets $\lfloor z\rfloor$. This is trivial if $x$ and $y$ are comparable, so let us assume they are incomparable. Let $w$ be the upper neighbour of $z$ such that $w \leqslant x$. Then $w \neq y$; let $x_{i}$ be the last vertex of $P$ with $w \leqslant x_{i}$. Then $x_{i+1} \in\lfloor z\rfloor$, because $T$ is normal.

Note that if $x \in T^{\prime}$ then $\lfloor x\rfloor \subset T^{\prime}$. Lemma 3.2 thus implies the following:

Lemma 3.3. If $P=x \ldots y$ is a $T^{\prime}-T^{\prime}$ path in $G$, then $x$ and $y$ are comparable in $S$.

Proof. If $x$ and $y$ are incomparable, then neither of them is in $\lfloor x\rfloor \cap\lfloor y\rfloor$, and so $\stackrel{\circ}{P} \cap\lfloor x\rfloor \cap\lfloor y\rfloor \neq \emptyset$ by Lemma 3.2. Since $\lfloor x\rfloor \cap\lfloor y\rfloor \subset T^{\prime}$, this means that $P$ is not a $T^{\prime}-T^{\prime}$ path.

Note that Lemma 3.2 does not imply that the interior of an $x-y$ path meets $\lfloor x\rfloor \cap\lfloor y\rfloor$. The $T^{\prime}-T^{\prime}$ path in Lemma 3.3, for example, may have a non-empty interior lying in $G \backslash T^{\prime}$ somewhere above $x$ and $y$.

Let $S$ be a subtree of $T$ rooted at $r$. (In applications, $S$ will be either a normal ray or equal to $T^{\prime}$.) Our next lemma says that any vertex of $S$ which is 'directly accessible' from some vertex $x \notin S$ (by a path in $G$ whose interior avoids $S$ ) must lie below $x$.

Lemma 3.4. Let $S$ be a subtree of $T$ rooted at $r$, and let $x \in V(G)$. Then $S[x] \subset\lfloor x\rfloor$. 
Proof. If $x \in S$, then $S[x]=\{x\} \subset\lfloor x\rfloor$. If $x \notin S$, let $P=x \ldots y$ be an $x$ $S$ path in $G$. By Lemma 3.2, $P$ meets $\lfloor x\rfloor \cap\lfloor y\rfloor \subset S$, while $\stackrel{\circ}{P} \cap S=\emptyset$ by the choice of $P$. Hence $P \cap\lfloor x\rfloor \cap\lfloor y\rfloor=\{y\}$, so $y \in\lfloor x\rfloor$ as required.

Lemma 3.4 implies that, if $x$ is a vertex and $R$ is a normal ray in $G$, the vertex set of $R[x]$ is a finite chain in $\leqslant_{T}$. Let us define the $R$-height of $x$, denoted by ht $(x ; R)$, to be the unique maximal vertex of $R[x]$. For example, the $R$-height of a vertex $h \in R$ is $h$ itself; the other vertices of $R$-height $h$ are precisely those vertices which lie above $h$ but not above any vertex of $h R$.

The concept of height will provide essential help in organizing our constructions of subgraphs of $G$ such as fan and bundle graphs. For $H \subset G$ or $H \subset V(G)$, we shall say that the $R$-height of (the vertices in) $H$ is bounded if $R$ has a vertex $h$ such that ht $(x ; R) \leqslant h$ for every vertex $x \in H$. Another subgraph $H^{\prime} \subset G$ (or $H^{\prime} \subset V(G)$ ) is $H$-clear with respect to $R$ if the $R$-height of each vertex of $H^{\prime}$ is strictly greater than the $R$-height of every vertex in $H$. Then, for example, if we are trying to find a fan graph in $G$ and $H$ is a partial fan graph constructed so far (on the path $P$, say), we shall aim to extend $P$ into $H^{\prime}$ and continue our construction there; we can then be sure that fans constructed at later stages will not interfere with earlier fans.

The first assertion in our next lemma is immediate from the definition of $R$-height. The second is a consequence of the fact that, by definition, the graph $R[x]$ coincides for all vertices $x$ in a common component of $G \backslash R$.

Lemma 3.5. Let $x, y \in G$, and let $R$ be a normal ray.

(i) If $y \in R[x]$, then $\operatorname{ht}(x ; R) \geqslant y=\operatorname{ht}(y ; R)$.

(ii) If $x$ and $y$ are in a common component of $G \backslash R$, then ht $(x ; R)=\operatorname{ht}(y ; R)$.

If $R$ and $R^{\prime}$ are normal rays, and if $x \in R \backslash R^{\prime}$ and $y \in R^{\prime} \backslash R$, then $R \cap R^{\prime}$ separates $x$ from $y$ in $G$ (by Lemma 3.2). This observation extends to any pair of vertices whose $R$-height and $R^{\prime}$-height, respectively, is sufficiently large:

Lemma 3.6. Let $R$ and $R^{\prime}$ be distinct normal rays; set $z:=\max \left(R \cap R^{\prime}\right)$. Suppose that $x, y \in V(G)$ are such that $\operatorname{ht}(x ; R)>z$ and $\operatorname{ht}\left(y ; R^{\prime}\right)>z$. Then $\lfloor z\rfloor=R \cap R^{\prime}$ separates $x$ from $y$ in $G$.

Proof. Write $u:=\operatorname{ht}(x ; R)$ and $v:=\operatorname{ht}\left(y ; R^{\prime}\right)$, and let $P$ be any $x-y$ path in $G$. Moreover, let $x \ldots u$ be an $x-R$ path and $y \ldots v$ a $y-R^{\prime}$ path in $G$. Since the walk $u \ldots x P y \ldots v$ meets $R \cap R^{\prime}$ (by Lemma 3.2) but neither $x \ldots u$ nor $y \ldots v$ meets $R \cap R^{\prime}$ (by definition), $P$ meets $R \cap R^{\prime}$ as claimed.

Our next lemma asserts that if the $R$-height of $x \notin R$ is sufficiently large, then its $R^{\prime}$-height is the same as that of the vertices on $R \backslash R^{\prime}$ itself, namely $z=\max \left(R \cap R^{\prime}\right)$. 
Lemma 3.7. Let $R$ and $R^{\prime}$ be distinct normal rays, $z:=\max \left(R \cap R^{\prime}\right)$, and $x \in V(G)$. If ht $(x ; R)>z$ then $\operatorname{ht}\left(x ; R^{\prime}\right)=z$.

Proof. Let $P$ be an $x-R$ path in $G$ whose last vertex $y$ is above $z$. Then $P y R z$ is an $x-\left(R \cap R^{\prime}\right)$ path, so ht $\left(x ; R^{\prime}\right) \geqslant z$ by definition. Now if $h t\left(x ; R^{\prime}\right)>z$ then Lemma 3.6, applied with $y=x$, would give that $\lfloor z\rfloor$ separates $x$ from $x$, a contradiction. Hence ht $\left(x ; R^{\prime}\right)=z$, as claimed.

If $R$ is a normal ray and $H$ is any subgraph of $G$, let us say that the $R$ height of $H$ tends to infinity if for each $h \in R$ only finitely many vertices of $H$ have $R$-height $\leqslant h$. Lemma 3.7 then has the following immediate consequence:

Lemma 3.8. If $R$ and $R^{\prime}$ are distinct normal rays, and if the $R$-height of $H \subset G$ tends to infinity, then the $R^{\prime}$-height of $H$ is bounded.

If $R$ is any ray in $G$ and $R^{\prime}$ is a normal ray, let us say that $R$ follows $R^{\prime}$ if $\left|R \cap R^{\prime}\right|=\infty$.

Lemma 3.9. Every ray in $G$ follows a unique normal ray.

Proof. Let $R \subset G$ be a ray. By Lemma 3.6, $R$ can follow at most one normal ray. We show that such a ray exists, by constructing a normal ray $x_{0} x_{1} \ldots$ which $R$ meets infinitely often. Let us assume that $R$ starts at the root $r$ of $T$; since this can be achieved by adding or deleting finitely many vertices of $R$, there will be no loss of generality.

Let $x_{0}:=r$. Suppose vertices $x_{0}, \ldots, x_{n}$ have been chosen so that they induce an increasing path in $T$ and infinitely many vertices of $R$ lie above $x_{n}$. Since $R$ can pass through $\left\lfloor x_{n}\right\rfloor$ only finitely often, Lemma 3.2 implies that $x_{n}$ has a unique upper neighbour $y$ in $T$ such that $R$ has infinitely many vertices above $y$; put $x_{n+1}:=y$.

In order to show that $R$ meets $x_{0} x_{1} \ldots$ infinitely often, suppose $x_{n}$ is the last vertex of $R$ that belongs to $\left\{x_{0}, x_{1}, \ldots\right\}$. (For the existence of such a vertex, recall that $x_{0}=r \in R$ by assumption.) Let $y$ be the next vertex on $R$, and let $m$ be the $r-y$ distance in $T$. Then $y$ and $x_{m}$ have the same distance from $r$ in $T$, and $y \neq x_{m}$. Hence $y$ and $x_{m}$ must be incomparable. By definition of $x_{m}, y R$ contains a vertex $x$ above $x_{m}$. By Lemma 3.2, then, $y R$ meets

$$
\lfloor x\rfloor \cap\lfloor y\rfloor \subset\left\lfloor x_{m}\right\rfloor=\left\{x_{0}, \ldots, x_{m}\right\},
$$

contrary to the choice of $x_{n}$.

Note that, since normal rays are contained in $T^{\prime}$, Lemma 3.9 implies in particular that $G \backslash T^{\prime}$ is rayless.

Our next lemma says that if a ray $R$ follows a normal ray $R^{\prime}$, then, in terms of $R^{\prime}$-height, the vertices of $R$ behave like those of $R^{\prime}$ itself: 
Lemma 3.10. Let $R \subset G$ be a ray, and let $R^{\prime}$ be the normal ray it follows. Then the $R^{\prime}$-height of $R$ tends to infinity.

Proof. Let $x \in R^{\prime}$ be given; we have to show that at most finitely many vertices of $R$ have $R^{\prime}$-height $\leqslant x$. Let $U:=\left\{v \in V(G) \mid \operatorname{ht}\left(v ; R^{\prime}\right) \leqslant x\right\}$. Then $\lfloor x\rfloor$ separates $\stackrel{x}{x} R^{\prime}$ from $U$; recall that $R^{\prime}[v] \subset\lfloor x\rfloor$ for $v \in U$, by definition of ht $\left(v ; R^{\prime}\right)$. Since $R$ meets $x R^{\prime}$ infinitely often, this implies that $V(R) \cap U$ is finite.

Our last lemma shows how vertices with neighbourhoods of unbounded $R$-height can be used to find a copy of $\mathrm{T} K_{\omega}$ in $G$.

Lemma 3.11. Let $R$ be a normal ray.

(i) If the neighbourhood of a vertex $x \in G$ has unbounded $R$-height, then $x$ is on $R$.

(ii) If $G$ has infinitely many vertices with neighbourhoods of unbounded $R$ height, then $G$ contains a $\mathrm{T} K_{\omega}$.

Proof. (i) If $x$ is not in $R$, then the $R$-height of each of its neighbours is at most its own $R$-height, by Lemma 3.5 .

(ii) Starting with $X_{0}:=\emptyset$, let us define inductively a nested sequence $X_{0} \subset X_{1} \subset \ldots$ of subgraphs of $G$ so that, for each $n, \quad X_{n} \simeq \mathrm{T} K_{n}$ and the neighbourhood of any branch vertex of $X_{n}$ has unbounded $R$-height. Let $n>0$, and suppose that $X_{n-1} \simeq \mathrm{T} K_{n-1}$ has already been defined. Let $x_{1}, \ldots, x_{n-1}$ be the branch vertices of $X_{n-1}$; the neighbourhoods of these vertices all have unbounded $R$-heights. Since $X_{n-1}$ is finite, $G \backslash X_{n-1}$ contains a vertex $x_{n}$ whose neighbourhood has unbounded $R$-height.

Let us define independent paths $P_{1}, \ldots, P_{n-1}$ joining $x_{n}$ to $x_{1}, \ldots, x_{n-1}$, respectively. Let $i \in\{1, \ldots, n-1\}$ be given, and assume that $P_{j}$ has been defined for all $j<i$. Let $y_{i}$ and $y_{n}$ be neighbours of $x_{i}$ and $x_{n}$, respectively, whose $R$-heights $z_{i}$ and $z_{n}$ exceed the $R$-heights of all the vertices in

$$
X_{n}^{i-1}:=X_{n-1} \cup \bigcup_{j<i} P_{j} .
$$

Let $y_{i} \ldots z_{i}$ be a $y_{i}-R$ path and $y_{n} \ldots z_{n}$ a $y_{n}-R$ path in $G$. Note that the vertices on these two paths have uniform $R$-heights of $z_{i}$ and $z_{n}$, respectively. Let $P_{i}$ be an $x_{i}-x_{n}$ path whose interior is contained in the walk $y_{i} \ldots z_{i} R z_{n} \ldots y_{n}$. Then the vertices of $\stackrel{\circ}{P}_{i}$ have $R$-heights between $z_{i}$ and $z_{n}$, so $\stackrel{\circ}{P}_{i}$ avoids $X_{n}^{i-1}$.

Putting

$$
X_{n}:=X_{n-1} \cup \bigcup_{i<n} P_{i},
$$

we see that $X_{n} \simeq \mathrm{T} K_{n}$ as intended. Moreover, the branch vertices of $X_{n}$ include those of $X_{n-1}$, and they all have neighbourhoods of unbounded $R$-height. This completes the induction step. It is clear that $\bigcup_{n \in \mathbb{N}} X_{n}$ is isomorphic to $\mathrm{T} K_{\omega}$. 


\section{Partitions of $T_{\omega}$}

In this section we collect for convenience two simple Ramsey-type lemmas, which will be needed repeatedly later.

Both our lemmas are about finding $\mathrm{T} T_{\omega}$ subtrees in a $\mathrm{T} T_{\omega}$. If $T \simeq \mathrm{T} T_{\omega}$, a colouring of $T$ will be any map whose domain is the set of branch vertices of $T$; the images of such a colouring are called colours. $T$ is monochromatic (or red, blue etc.) if all its branch vertices are coloured by (mapped to) the same colour (red, blue etc.), and $T$ is injectively coloured if no two of its branch vertices have the same colour.

The proof of our first lemma is straightforward.

Lemma 4.1. If $T$ is a rooted $T T_{\omega}$ with a red-blue colouring, then $T$ contains either a red $\mathrm{T} T_{\omega}$ of the form $T^{\geqslant v}$ (for some branch vertex $v$ of $T$ ) or a blue $\mathrm{T} T_{\omega}$ (whose minimal vertex is one of its branch vertices).

As a point of reference, let $T^{*}$ be a fixed copy of a rooted $T_{\omega}$, given with an enumeration $e_{1}^{*}, e_{2}^{*}, \ldots$ of its edges such that, for each $n \in \mathbb{N}$, the graph $T_{n}^{*} \subset T^{*}$ consisting of the root of $T^{*}$ and the edges $e_{1}^{*}, \ldots, e_{n}^{*}$ (together with their endvertices) is connected. Such an enumeration clearly exists; for example, one could first list the edges of $T^{*}$ using any enumeration, and then assemble $T^{*}$, starting from the root, by adding at each of $\omega$ steps the minimal edge from the list that keeps the covered portion of $T^{*}$ connected. The $n$th edge $e$ on the list will then be added after no more than $k(n)$ steps, where $k(n)$ is the sum of the list positions of all the edges on the path $P$ in $T^{*}$ from the root to the far endvertex of $e$ (induction on the length of $P$ ).

We shall use this standard construction of $T^{*}$ in the proof of Lemma 4.2 below, and again in $\S 6$. Note that, for each $n$, exactly one of the two endvertices of $e_{n}^{*}$ is in $T_{n-1}^{*}$. This vertex will be denoted by $x_{n}^{*}$, the other endvertex of $e_{n}^{*}$ by $y_{n}^{*}$.

Lemma 4.2. Any coloured rooted $\mathrm{T} T_{\omega}$ contains either a monochromatic $\mathrm{T} T_{\omega}$ or an injectively coloured $\mathrm{TT}_{\omega}$, whose minimal vertex (in either case) is among its branch vertices.

Proof. Let $T$ be a coloured $T T_{\omega}$. Assume first that the colouring of $T$ has the following property:

(4.3) For each vertex $v \in T$, the subtree $T^{\geqslant v}$ uses infinitely many colours.

Following the standard construction of $T^{*}$, we build up an injectively coloured $\mathrm{T} T_{\omega}=: T^{\prime} \subset T$, say with branch vertices $x_{n}$ and $y_{n}$ corresponding to the vertices $x_{n}^{*}$ and $y_{n}^{*}$ of $T^{*}$, as follows. We start by picking any branch vertex of $T$ as $x_{1}$. Later, whenever we come to choose a subdivided edge $x_{n} \ldots y_{n}$ of $T^{\prime}$ corresponding to an edge $e_{n}^{*}$ of $T^{*}$, we first select an upper neighbour $v$ of $x_{n}$ 
that does not lie in the (finite) part of $T^{\prime}$ constructed so far. We then use (4.3) to choose $y_{n}$ among the branch vertices of $T^{\geqslant v}$ so that its colour differs from the (finitely many) colours used by branch vertices of $T^{\prime}$ selected earlier, and let $x_{n} \ldots y_{n}$ be the unique $x_{n}-y_{n}$ path in $T$.

Suppose now that (4.3) fails, i.e. that $T$ has a vertex $v$ such that $T^{\geqslant v}$ uses only finitely many colours. Without loss of generality, $v$ is a branch vertex. We may now obtain a monochromatic $\mathrm{T} T_{\omega}$ inside $T^{\geqslant v}$ by repeated application of Lemma 4.1: we just partition the colours used into two classes called 'red' and 'blue', apply the lemma and iterate.

For a thorough study of Ramsey-type properties of $T_{\omega}$, the reader is referred to Prömel and Voigt [8].

\section{Good families}

In our introductory discussion of the three countable types of forbidden subgraph in the bounded graph conjecture, the $\mathrm{T} T_{\omega}$, the bundle graph, and the fan graph, we noticed that the proofs of these graphs' own unboundedness were very similar. In the case of $T_{\omega}$, we just took any injective labelling and observed that, for any given sequence $\sigma: \mathbb{N} \rightarrow \mathbb{N}$, we could easily find a ray $R$ through $T_{\omega}$ which was not dominated by $\sigma$. Indeed, no matter how we had chosen an initial segment of $R$, we would have an infinite choice of labels for the next vertex of $R$, which could thus be chosen larger than the corresponding term in $\sigma$.

For a bundle graph or a fan graph, finding such a ray $R$ was hardly more difficult. All we had to make sure of was that the paths $P$ we considered for initial segments of $R$ belonged to a certain family $\mathcal{P}$ : a family of paths which could, again and again, be extended to reach unused vertices of infinite degree. In the bundle graph $B, \mathcal{P}$ consisted of the paths from left to right; in the fan graph $F$, of those towards the right and down the spines of the fans.

The idea of finding such a family of paths in an unbounded graph is central to our proof of the bounded graph conjecture. Our aim in this section, essentially, is to prove that in every unbounded graph such a family of paths exists. In the next section we shall show that one can always reconstruct one of the forbidden subgraphs from the paths in that family.

Let us make precise the kind of family of paths in which we are interested, so that we can state exactly what we shall prove in this section. Let $G$ be an arbitrary connected graph with a normal spanning tree $T$, and let $T^{\prime} \subset T$ be the union of all normal rays in $G$. Call a pair $(x, y)$ of distinct vertices of $T^{\prime}$ good if $G$ contains only finitely many $T^{\prime}-T^{\prime}$ paths from $x$ to $y$, and bad otherwise. A non-empty family $\mathcal{P}$ of finite paths in $G$ will be called a good family if it satisfies the following four conditions: 
(i) for every path $P \in \mathcal{P}$ there exists an $n \in \mathbb{N}$ such that $P$ has infinitely many extensions in $\mathcal{P}$ of length $n$;

(ii) if $P \in \mathcal{P}, x, y \in V(P)$, and $x P y$ is a $T^{\prime}-T^{\prime}$ path in $G$ of length $\geqslant 2$ (i.e. with at least 2 edges), then $(x, y)$ is a good pair;

(iii) every path $P \in \mathcal{P}$ starts at the root of $T$;

(iv) if $P \in \mathcal{P}$ and $Q$ is an initial segment of $P$, then $Q \in \mathcal{P}$.

Note that the essence of a good family is contained in condition (i): if we choose $n$ minimal, then infinitely many extensions $P^{\prime} \in \mathcal{P}$ of $P$ agree up to their $n$th (= penultimate) vertex and differ pairwise at their last vertex. Thus, if $P$ is viewed as the beginning of a ray $R$ being constructed to elude a given sequence $\sigma$ (as in our discussion above), then the $(n+1)$ th vertex of this ray can be selected from an infinite choice, and therefore in such a way that its label exceeds the corresponding term in $\sigma$. (So, by condition (i) alone, the existence of a good family of paths in $G$ implies that $G$ is unbounded.)

Condition (ii) says, less formally, that paths of a good family leave and return to $T^{\prime}$ only at good pairs of vertices. This assumption is also vital to our proof: it ensures that subgraphs constructable from the paths in a good family have many vertices on $T^{\prime}$-for example, we shall see that any vertex of infinite degree has to be on $T^{\prime}$-which will give us the amount of control over these subgraphs that we shall need.

Conditions (iii) and (iv) are normalization conditions; they are not essential, but they will make it easier to handle good families.

Recall that, if $\kappa$ is a cardinal, $I_{\kappa}$ denotes the disjoint union of $\kappa$ rays. Let us define $\mathfrak{b}$ to be the least cardinal $\kappa$ such that there exists a family of $\kappa$ distinct $\mathbb{N} \rightarrow \mathbb{N}$ sequences which are not dominated simultaneously by any $\mathbb{N} \rightarrow \mathbb{N}$ sequence. (This cardinal $\mathfrak{b}$ is commonly known as the bounding number). Then, in particular, every family of fewer than $\mathfrak{b}$ sequences has a majorant. Note also that $\omega<\mathfrak{b} \leqslant 2^{\omega}$ by Lemma 1.1 .

As is easy to check, $\mathfrak{b}$ is regular, i.e. $\mathfrak{b}$ is not the union (sum) of fewer than $\mathfrak{b}$ cardinals $<\mathfrak{b}$. Moreover, since $I_{\kappa}$ contains exactly $\omega \cdot \kappa=\kappa$ rays whenever $\kappa$ is an infinite cardinal, $\mathfrak{b}$ is also the least cardinal such that $I_{\mathfrak{b}}$ is unbounded.

We can now state the theorem which we shall prove in this section.

Theorem 5.1. Let $G$ be an unbounded graph with a normal spanning tree, and suppose that $G$ contains neither an $I_{\mathfrak{b}}$ nor a bundle graph. Then there exists a good family of paths in $G$.

Proof. As usual, let the normal spanning tree of $G$ be denoted by $T$, and the union of all normal rays in $G$ by $T^{\prime}$.

Let us show straight away that

$$
\left|T^{\prime}\right|<\mathfrak{b} .
$$


Since $T^{\prime}$ is connected and $\mathfrak{b}$ is regular, it suffices to show that each vertex $v \in$ $T^{\prime}$ has degree $<\mathfrak{b}$ in $T^{\prime}$. Let $W$ be the set of upper neighbours of $v$ in $T^{\prime}$ and, for each $w \in W$, let $R_{w}$ be any normal ray containing $w$. Then the tails $w R_{w}$ for $w \in W$ are pairwise disjoint. Since $G \nsupseteq I_{\mathfrak{b}}$ by assumption, this means that $v$ has degree $<\mathfrak{b}$, as claimed.

The proof of the theorem is organized as follows. We begin by constructing a family $\mathcal{P}$ of paths in $G$ which satisfies the four defining conditions of a good family, but which will not, a priori, be known to be non-empty. Such a family is easily obtained: we shall start with the family $\mathcal{P}_{0}$ of all paths in $G$ which have any chance of eventually being in $\mathcal{P}$ (omitting, for example, any paths which violate condition (ii)), and then recursively discard those paths from this family which fail to meet the extendability condition (i).

The main part of the proof will be to show that $\mathcal{P}$ is indeed non-empty and therefore a good family. The key here will be that the recursion by which $\mathcal{P}$ is obtained takes fewer than $\mathfrak{b}$ steps. At each step $\alpha$, we shall find a sequence $\sigma_{\alpha}: \mathbb{N} \rightarrow \mathbb{N}$ which dominates all those rays in $G$ whose initial segments were all in $\mathcal{P}_{0}$, and for which some initial segment was discarded at step $\alpha$. Then, by the definition of $\mathfrak{b}$, there will be a sequence $\sigma$ which dominates all such rays in $G$ simultaneously. Since $G$ is by assumption unbounded, and therefore contains a ray $R$ which is not dominated by $\sigma$, this means that the initial segments of such a ray $R$ (after some normalization) are either still in $\mathcal{P}$ when the recursion ends, or were not all in $\mathcal{P}_{0}$ at the start of the recursion. In the former case $\mathcal{P}$ will be non-empty, as desired. In the latter case, it will turn out that $R$ leaves and returns to $T^{\prime}$ infinitely often at bad pairs $(x, y)$; we shall then be able to construct a bundle graph in $G$ along $R$ (typically with the bundles occurring between these $x$ and $y$ ), in contradiction to our assumptions.

Let us define $\mathcal{P}$ precisely. Let $\mathcal{Q}_{0}$ be the set of all paths $P \subset G$ starting at the root of $T$ and ending on $T^{\prime}$ which only leave $T^{\prime}$ at good pairs of vertices, i.e., which have the property that if $x P y$ is a $T^{\prime}-T^{\prime}$ path in $G$ of length $\geqslant 2$ then $(x, y)$ is a good pair. Let $\mathcal{P}_{0}$ be the set of all initial segments of paths in $\mathcal{Q}_{0}$; note that $\mathcal{P}_{0}$ includes $\mathcal{Q}_{0}$. Then $\mathcal{P}_{0}$ satisfies conditions (ii), (iii) and (iv) from the definition of a good family.

We claim that $\left|\mathcal{P}_{0}\right|<\mathfrak{b}$. As clearly $\left|\mathcal{P}_{0}\right|=\left|\mathcal{Q}_{0}\right|$, it suffices to show that $\left|\mathcal{Q}_{0}\right|<\mathfrak{b}$. Now, each path $P \in \mathcal{Q}_{0}$ is determined by its (finite) sequence of vertices on $T^{\prime}$ and the choice of its subpaths $x P y$ for successive $x, y \in V(P) \cap V\left(T^{\prime}\right)$. As $\left|T^{\prime}\right|<\mathfrak{b}$, there are only $<\mathfrak{b}$ finite sequences of vertices of $T^{\prime}$, and since each $x P y$ has length $\geqslant 2$ only if $(x, y)$ is good, the choice of subpaths $x P y$ is finite for each pair $(x, y)$. Hence $\left|\mathcal{Q}_{0}\right|<\mathfrak{b}$, as required.

For all ordinals $\alpha>0$, let us inductively define subsets $\mathcal{P}_{\alpha}$ of $\mathcal{P}_{0}$, as follows. Suppose first that $\alpha$ is a successor, say $\alpha=\beta+1$. If $\mathcal{P}_{\beta}$ contains a path $P=: P_{\beta}$ which violates (i) in the definition of a good family (i.e., a path $P$ such that, for each $n \in \mathbb{N}, \mathcal{P}_{\beta}$ contains only finitely many extensions of $P$ of length $n$ ), let $\mathcal{P}_{\alpha}$ be obtained from $\mathcal{P}_{\beta}$ by deleting $P_{\beta}$ and all its extensions $P^{\prime} \in \mathcal{P}_{\beta}$. If 
$\mathcal{P}_{\beta}$ contains no such path $P$, let $\mathcal{P}_{\alpha}:=\mathcal{P}_{\beta}$. If $\alpha$ is a limit, let $\mathcal{P}_{\alpha}:=\bigcap_{\beta<\alpha} \mathcal{P}_{\beta}$.

Since $\left|\mathcal{P}_{0}\right|<\mathfrak{b}$, there exists an $\alpha<\mathfrak{b}$ such that $\mathcal{P}_{\alpha+1}=\mathcal{P}_{\alpha}$; let $\alpha^{*}$ be the least such $\alpha$, and set $\mathcal{P}_{\alpha^{*}}=: \mathcal{P}$. Since each $\mathcal{P}_{\alpha}$ satisfies conditions (ii), (iii) and (iv) from the definition of a good family, and since $\mathcal{P}=\mathcal{P}_{\alpha^{*}}$ in addition satisfies condition (i), we see that $\mathcal{P}$ is a good family if and only if it is nonempty. To complete the proof, then, it suffices to show that if $\mathcal{P}$ is empty then $G$ contains a bundle graph, contrary to our assumption.

Let us suppose that $\mathcal{P}$ is indeed empty. We shall first prove that $G$ contains a ray $R$ which leaves $T^{\prime}$ infinitely often at bad pairs $(x, y)$, and then use $R$ to find a bundle graph in $G$.

(5.3) There exists a ray $R \subset G$ which contains infinitely many $T^{\prime}-T^{\prime}$ paths $x R y$ such that $(x, y)$ is a bad pair of vertices. ${ }^{*}$

To prove (5.3), let us suppose for a contradiction that any ray in $G$ leaves $T^{\prime}$ only finitely often at bad pairs of vertices. We show that $G$ is bounded, contrary to our assumptions.

Let $f: V(G) \rightarrow \mathbb{N}$ be an arbitrary labelling of $G$. We shall define sequences $\sigma_{\alpha}: \mathbb{N} \rightarrow \mathbb{N}$, one for each $\alpha<\alpha^{*}$, whose majorant $\sigma^{*}$ will bound $G$. For each $\alpha<\alpha^{*}$, let the $k$ th term of $\sigma_{\alpha}$ be defined as

$$
\sigma_{\alpha}(k):= \begin{cases}f\left(x_{k}\right) & \text { if } k \leqslant\left|P_{\alpha}\right| \\ \max \left\{f(v(P)) \mid P \in \mathcal{P}_{\alpha} \backslash \mathcal{P}_{\alpha+1} \text { and }|P|=k\right\} & \text { if } k>\left|P_{\alpha}\right|\end{cases}
$$

where $x_{k}$ is the $k$ th vertex of $P_{\alpha}$ and $v(P)$ is the last vertex of $P$. Recall that, by definition, $P_{\alpha}$ has only finitely many extensions $P \in \mathcal{P}_{\alpha}$ of any given length; since $\mathcal{P}_{\alpha} \backslash \mathcal{P}_{\alpha+1}$ is precisely the set of $P_{\alpha}$ and all its extensions in $\mathcal{P}_{\alpha}$, the maximum used above is therefore just the maximum of a finite set. Finally, let $\sigma^{*}$ be the majorant of $\left\{\sigma_{\alpha} \mid \alpha<\alpha^{*}\right\}$; it exists, because $\alpha^{*}<\mathfrak{b}$.

To show that $\sigma^{*}$ bounds $G$, let $R \subset G$ be any ray. By assumption, $R$ leaves $T^{\prime}$ only finitely often at bad pairs of vertices; let $R^{\prime}$ be a tail of $R$ which leaves $T^{\prime}$ only at good pairs. (Recall that since $R$ follows a normal ray (Lemma 3.9), it never leaves $T^{\prime}$ altogether.) Let $Q$ be a path in $T^{\prime}$ from the root of $T$ to $R^{\prime}$, and let $v$ be the last vertex of $Q$. Then the ray $R^{\prime \prime}:=Q v R^{\prime}(=Q v R)$ still leaves $T^{\prime}$ at good pairs only, so every initial segment of $R^{\prime \prime}$ is in $\mathcal{P}_{0}$.

Now since $\mathcal{P}=\emptyset$ by assumption, each initial segment of $R^{\prime \prime}$ is discarded at some (non-limit) step in the recursive definition of $\mathcal{P}$; let $\alpha<\alpha^{*}$ be minimal such that $\mathcal{P}_{\alpha} \backslash \mathcal{P}_{\alpha+1}$ contains an initial segment of $R^{\prime \prime}$. Then $P_{\alpha}$ is itself an initial segment of $R^{\prime \prime}$. Moreover, all the extensions of $P_{\alpha}$ in $R^{\prime \prime}$ are also in $\mathcal{P}_{\alpha}$ (by the minimality of $\alpha$ ), and were hence discarded together with $P_{\alpha}$. Thus,

\footnotetext{
* As the reader may expect (and the proof of (5.3) will indeed show), the paths $x$ Ry can be chosen so as to have lengths $\geqslant 2$. However, this will not be needed when (5.3) is used below.
} 
$\mathcal{P}_{\alpha} \backslash \mathcal{P}_{\alpha+1}$ contains every initial segment of $R^{\prime \prime}$ of length $k \geqslant\left|P_{\alpha}\right|$. Therefore $\sigma_{\alpha}$ dominates $R^{\prime \prime}$, by definition of $\sigma_{\alpha}$.

By Lemma 2.2, this implies that $\sigma^{*}$ dominates $R$, so $\sigma^{*}$ bounds $G$ as claimed. As this contradicts our assumption that $G$ is unbounded, we have thus completed the proof of (5.3).

Let $R \subset G$ be a ray as in (5.3). Let $\left(x_{1}, y_{1}\right),\left(x_{2}, y_{2}\right), \ldots$ be an infinite sequence of disjoint bad pairs of vertices, such that $x_{i}$ precedes $y_{i}$ on $R$ for each $i$ and $y_{i}$ precedes $x_{j}$ on $R$ for $i<j$. For each $i$, let $H_{i}$ be the union of all $T^{\prime}-T^{\prime}$ paths in $G$ from $x_{i}$ to $y_{i}$; note that, since $\left(x_{i}, y_{i}\right)$ is bad, $H_{i}$ is infinite. We remark that the graphs $H_{i}$ are not necessarily disjoint, and that $H_{i}$ may well meet $R$ even outside the segment $x_{i} R y_{i}$.

For each $i$, let us find a bundle $B_{i} \subset H_{i}$, say from a vertex $x_{i}^{\prime}$ to a vertex $y_{i}^{\prime}$, together with (possibly trivial) disjoint paths $C_{i}=x_{i} \ldots x_{i}^{\prime}$ and $C_{i}^{\prime}=y_{i}^{\prime} \ldots y_{i}$ in $H_{i}$ which avoid the interior of $B_{i}$. (These paths will be called the connectors of $B_{i}$; Fig. 4.)

FIgURE 4. The bundles $B_{i}$ and their connectors

To define $B_{i}$ for a given $i$, recall that since $G \backslash T^{\prime}$ is rayless (Lemma 3.9), $H_{i}$ too is rayless. Since $H_{i}$ is infinite and connected, it has a vertex $v$ of infinite degree, by König's theorem. As $H_{i}$ is the union of $x_{i}-y_{i}$ paths, each of the edges $e=u v$ incident with $v$ is contained in such a path $P(e)$. Now if $v$ precedes $u$ on $P(e)$ for infinitely many of these edges $e$ then, by Lemma 2.3, the union of their paths $P(e)$ contains a $v-w$ bundle $B$ (for some $w$ ) on the segment $v P$ of one of these paths $P$. (Lemma 2.3 does not give a fan, because $H_{i}$ is rayless.) Put $C_{i}:=P v$ and $C_{i}^{\prime}:=w P$ in this case, and let $B_{i}$ be obtained from $B$ by deleting those fibres which meet $P v$. On the other hand, if $u$ precedes $v$ on $P(e)$ for infinitely many of the edges $e$, then the union of the corresponding paths $P(e)$ contains a $w-v$ bundle $B$ (for some $w$ ) on the segment $P v$ of one of these paths $P$ (apply Lemma 2.3 'backwards'). In that case, we let $C_{i}:=P w$ and $C_{i}^{\prime}:=v P$, and let $B_{i}$ be obtained from $B$ by deleting those fibres which meet $v P$. (This construction of a bundle in a graph such as $H_{i}$ is due to Halin [5].)

Now that we have defined an infinite sequence of bundles along our ray $R$, it may seem that obtaining a bundle graph from them would be only a small step away. As remarked earlier, however, the subgraphs $H_{i}$ containing our bundles need not be disjoint. In fact, it might even happen that two of the 
bundles, as well as their connectors, coincide (except for the two vertices $x_{i}$ and $\left.y_{i}\right)$.

We shall deal with this problem as follows. By Lemma 3.9, $R$ follows some normal ray $R^{\prime}$. A natural way to ensure that different bundles $B_{i}$ are disjoint, therefore, is to separate them by their $R^{\prime}$-height: if we can find an infinite subsequence $i_{1}, i_{2}, \ldots$ spaced out sufficiently that each $B_{i_{k}}$ is $B_{i_{j}}$-clear with respect to $R^{\prime}$ for every $j<k$, then these bundles $B_{i_{k}}$ will indeed define a bundle graph along $R$. For this approach to work it is essential, of course, that the $R^{\prime}$-height of each bundle $B_{i_{k}}$ be bounded. Unfortunately, this is not necessarily the case - so let us deal with this problem first.

Suppose there are infinitely many $i$ such that the $R^{\prime}$-height of $B_{i}$ is unbounded. Since $\left(H_{i} \backslash\left\{x_{i}, y_{i}\right\}\right) \cap T^{\prime}=\emptyset$, every fibre of $B_{i}$ is disjoint from $R^{\prime}$, and so the vertices of each individual fibre have constant $R^{\prime}$-height. If the $R^{\prime}$-height of $B_{i}$ is unbounded, this implies that $x_{i}^{\prime}$ has a neighbourhood of unbounded $R^{\prime}$ height, and hence that $x_{i}^{\prime}=x_{i}$ (Lemma $3.11(\mathrm{i})$ ). If this happens for infinitely many $i$, the corresponding vertices $x_{i}$ - which are all distinct - form an infinite set of vertices each with a neighbourhood of unbounded $R^{\prime}$-height. Then $G$ contains a $\mathrm{T} K_{\omega}$ (and hence a bundle graph) by Lemma 3.11 (ii).

We may therefore assume from now on:

(5.4) The $R^{\prime}$-height of $B_{i}$ is bounded for all but finitely many $i$.

Using $R$, the bundles $B_{i}$ and their connectors $C_{i}$ and $C_{i}^{\prime}$, let us construct a bundle graph in $G$ inductively, adding one bundle at a time. More formally, let us construct by induction on $n$ a nested sequence $\left(G_{n}\right)_{n \in \mathbb{N}}$ of graphs with the following properties:

(i) $G_{n}$ is a partial bundle graph (with $n$ bundles), on a path $P_{n}$ which ends in a vertex $t_{n} \in R$;

(ii) $P_{n}$ has a vertex $s_{n} \neq t_{n}$ such that all the bundles of $G_{n}$ are between vertices preceding $s_{n}$ on $P_{n}$;

(iii) $s_{n} P_{n} \subset R$, and $s_{n}$ precedes $t_{n}$ on $R$;

(iv) $t_{n} R$ is $S_{n}$-clear with respect to $R^{\prime}$, where $S_{n}:=G_{n} \backslash \stackrel{s}{s}_{n} P_{n}$.

(See Figure 5.)

Figure 5. The partial bundle graph $G_{n}$ 
Condition (iv) means that every vertex on $R$ from $t_{n}$ onwards has $R^{\prime}$-height strictly greater than the $R^{\prime}$-height of any vertex in $G_{n}$ up to $s_{n}$. So in particular, the $R^{\prime}$-height of $S_{n}$, and hence that of $G_{n}=S_{n} \cup s_{n} R t_{n}$, is bounded:

$G_{n}$ has bounded $R^{\prime}$-height.

To define $G_{0}$, let $s_{0}$ be the first vertex of $R$, and put $G_{0}:=P_{0}:=R t_{0}$ where $t_{0} \in R$ is chosen so that $t_{0} R$ is $\left\{s_{0}\right\}$-clear with respect to $R^{\prime}$; this can be done by Lemma 3.10 (which we shall use freely in our further construction). Suppose now we have defined $G_{k}$, for $k=0, \ldots, n$, in accordance with conditions (i)-(iv). We shall now define $G_{n+1}$.

By (5.4) and (5.5), choose $i \in \mathbb{N}$ so that $B_{i}$ has bounded $R^{\prime}$-height and $x_{i} R$ is $G_{n}$-clear with respect to $R^{\prime}$. Note that $t_{n} R x_{i}$ meets $G_{n}$ only in $t_{n}$ : it avoids $S_{n}$ by (iv), and it meets $s_{n} R t_{n}$ only in $t_{n}$, because $s_{n}$ precedes $t_{n}$ on $R$ by (iii) and $t_{n}$ precedes $x_{i}$ on $R$ by the choice of $x_{i}$. Note that also

$$
H_{i}^{\prime}:=\left(C_{i} \cup B_{i} \cup C_{i}^{\prime}\right) \backslash\left\{x_{i}, y_{i}\right\}
$$

is $G_{n}$-clear with respect to $R^{\prime}$ : since each $v \in H_{i}^{\prime}$ can be connected to $x_{i}$ by a $v-T^{\prime}$ path, the $R^{\prime}$-height of $v$ is at least that of $x_{i}$ (Lemma 3.5).

Using our assumption that $B_{i}$ (and hence $H_{i}^{\prime}$ ) has bounded $R^{\prime}$-height, choose $u \in y_{i} R$ so that $u R$ is $X$-clear with respect to $R^{\prime}$, where

$$
X:=G_{n} \cup H_{i}^{\prime}
$$

Let $B_{i}^{\prime}$ be obtained from $B_{i}$ by removing those fibres that have a vertex on $t_{n} R u$; as there are at most finitely many such fibres, $B_{i}^{\prime}$ is still a bundle. The interior of $B_{i}^{\prime}$ then avoids $t_{n} R u$, and it also avoids $G_{n}$ (because $H_{i}^{\prime}$ does, and $H_{i}^{\prime}$ contains the interior of $B_{i}^{\prime}$ ).

The connectors $C_{i}$ and $C_{i}^{\prime}$, however, may still meet $t_{n} R x_{i}$ or $y_{i} R u$. Let $x_{i}^{\prime \prime}$ be the last vertex on $C_{i}$ that is in $t_{n} R u$, and let $y_{i}^{\prime \prime}$ be the first vertex on $C_{i}^{\prime}$ that is in $t_{n} R u$ (Fig. 6). Rename $x_{i}^{\prime \prime}$ and $y_{i}^{\prime \prime}$ as $a$ and $b$, where $a$ precedes $b$ on $R$, and set

$$
U:=G_{n} \cup t_{n} R a \cup\left(x_{i}^{\prime \prime} C_{i} x_{i}^{\prime} \cup B_{i}^{\prime} \cup y_{i}^{\prime} C_{i}^{\prime} y_{i}^{\prime \prime}\right) \cup b R u .
$$

Then $U$ is a partial bundle graph, with $n+1$ bundles, whose $R^{\prime}$-height is bounded.

In order to complete the induction step formally, set $s_{n+1}:=u$, and choose $t_{n+1} \in u R$ so that $t_{n+1} R$ is $U$-clear with respect to $R^{\prime}$. Note that $u R t_{n+1} \cap U=\{u\}$, because $U \subset X \cup R u$ and $u R t_{n+1}$ is $X$-clear by the choice of $u$. Set $G_{n+1}:=U \cup u R t_{n+1}$, and let

$$
P_{n+1}:=P_{n} \cup t_{n} R a \cup\left(x_{i}^{\prime \prime} C_{i} x_{i}^{\prime} \cup F \cup y_{i}^{\prime} C_{i}^{\prime} y_{i}^{\prime \prime}\right) \cup b R t_{n+1},
$$


Figure 6. Disentangling $R$ and $H_{i}^{\prime}$

where $F$ is some $x_{i}^{\prime}-y_{i}^{\prime}$ path in $B_{i}^{\prime}$. Then $G_{n+1}$ is a partial bundle graph on $P_{n+1}$, satisfying conditions (i)-(iv) for $n+1$.

The graph $\bigcup_{n \in \mathbb{N}} G_{n}$, finally, is a bundle graph as desired. This completes the proof of Theorem 5.1.

\section{Fans and bundles from a good family tree}

This section is devoted to the proof of the following theorem.

Theorem 6.1. Let $G$ be a graph with a normal spanning tree, and suppose there exists a good family of paths in $G$. Then $G$ contains a $T T_{\omega}$, a bundle graph, or a fan graph.

Proof. As before, let the normal spanning tree of $G$ be denoted by $T$, and the union of all normal rays by $T^{\prime}$. Let $\mathcal{P}$ be a good family of paths in $G$. The method of our proof is as follows.

We shall represent the structure of $\mathcal{P}$ by a certain tree $\mathcal{T}$, which will act as something like a covering space for the paths $P \in \mathcal{P}$ in $G$. Thus, the paths of $\mathcal{P}$ will 'lift' to paths in $\mathcal{T}$, which will be arranged in such a way as to reflect the relation of extension between their projections in $\mathcal{P}$.

Our main task will be to study the relationship between $\mathcal{T}$ and $G$. If $G$ (more precisely, the subgraph $\bigcup \mathcal{P}$ of $G$ ) resembles $\mathcal{T}$ closely, we shall try to find a tree of type $\mathrm{TT}_{\omega}$ in $G$. On the other hand, if the paths of $\mathcal{P}$ intersect a lot more (in $G$ ) than do their lifts in $\mathcal{T}$, we shall seek to construct a bundle graph or a fan graph in $G$. The distinction between the latter two cases will depend on the relationship between $\mathcal{T}$ and our normal spanning tree $T$ of $G$.

Recall that the essence of the fact that $\mathcal{P}$ is a good family of paths lies in its extension property, condition (i) of the definition of a good family: for each of its paths, $\mathcal{P}$ contains infinitely many extensions of some common length. We start by 'pruning' $\mathcal{P}$ to a subfamily containing no more paths than the extension property requires. 
To be precise, let us define subsets $\mathcal{Q}_{0}, \mathcal{Q}_{1}, \ldots$ of $\mathcal{P}$ as follows. Pick any non-trivial path $P_{0} \in \mathcal{P}$, and set $\mathcal{Q}_{0}:=\left\{P_{0}\right\}$. Having defined $\mathcal{Q}_{0}, \ldots, \mathcal{Q}_{n}$, we want $\mathcal{Q}_{n+1}$ to be a set of extensions of the paths in $\mathcal{Q}_{n}$ just large enough to witness the fact that $\mathcal{Q}_{n} \subset \mathcal{P}$. For each $P \in \mathcal{Q}_{n}$, take the minimal $k$ such that $P$ has infinitely many extensions of length $k$ in $\mathcal{P}$. Then infinitely many of these extensions agree up to their penultimate vertices: recall that $\mathcal{P}$ is closed under taking initial segments. We may thus choose infinitely many extensions $P^{(0)}, P^{(1)}, \ldots$ of $P$ in $\mathcal{P}$, each of length $k$, which differ only in their last vertices. Set

$$
\mathcal{Q}_{n+1}:=\left\{P^{(i)} \mid P \in \mathcal{Q}_{n}, i \in \mathbb{N}\right\},
$$

and continue inductively. If we now let $\mathcal{Q}$ be the set of all initial segments of paths in $\bigcup_{n \in \mathbb{N}} \mathcal{Q}_{n}$, we see that $\mathcal{Q}$ is a good family.

In structure, $\mathcal{Q}$ is rather similar to a $\mathrm{T} T_{\omega}$. Let us make this precise. Call a graph $H$ a long-rooted $\mathrm{TT}_{\omega}$ if $H$ is a subdivision of a rooted tree whose root $r$ has degree 1 and all of whose other vertices have countably infinite degree. The edge incident with $r$ in this tree then corresponds to a path $P$ in $H$, which will be called the long root of $H$. (Thus, $P$ is the unique path $r \ldots x \subset H$ for which $H \backslash P \stackrel{\circ}{\mathrm{x}} \simeq \mathrm{T} T_{\omega}$.) To avoid confusion, we may refer to $r$ itself as the root vertex of $H$.

Now define a tree $\mathcal{T}$ as follows. The vertex set of $\mathcal{T}$ is $\mathcal{Q}$ itself. For $P, P^{\prime} \in \mathcal{Q}$, we join $P$ to $P^{\prime}$ by an edge whenever $P^{\prime}$ is an extension of $P$ with $\left|P^{\prime}\right|=|P|+1$, i.e. if $P$ may be obtained from $P^{\prime}$ by removing its last vertex. Then $\mathcal{T}$ is a long-rooted $T T_{\omega}$, whose root vertex $\phi$ is the trivial path consisting of the first vertex of $P_{0}$ (which in turn is the root of $T$ ). Note that, for $\alpha, \beta \in V(\mathcal{T})$, we have $\alpha \leqslant_{\mathcal{T}} \beta$ if and only if $\alpha$ is an initial segment of $\beta$.

Any long-rooted $\mathrm{TT}_{\omega}$ contained in $\mathcal{T}$ and also rooted at $\phi$ will be called a good family tree. Clearly, the vertex set of such a good family tree is again a good family of paths in $G$. In fact, the only property of $\mathcal{T}$ we shall ever use is that it is a good family tree: we shall repeatedly prune $\mathcal{T}$ to a smaller good family tree $\mathcal{T}^{\prime}$, and then rename $\mathcal{T}^{\prime}$ as $\mathcal{T}$.

For $\alpha \in V(\mathcal{T})$, let $v(\alpha)$ denote the last vertex of the path $\alpha$. Note that if $\beta$ is another vertex of $\mathcal{T}$ and $\beta<\mathcal{T} \alpha$, then the path $\beta \subset G$ is a proper initial segment of the path $\alpha$, and so $v(\beta) \neq v(\alpha)$. Likewise, $v(\alpha)$ and $v(\beta)$ will be distinct if the paths $\alpha$ and $\beta$ were picked for $\mathcal{Q}$ at the same step $n$, and as extensions $P^{(i)}$ of the same path $P \in \mathcal{Q}_{n-1}$. We may therefore note the following:

(6.2) If $\mathcal{T}^{\prime}$ is a good family tree, and if $\alpha, \beta \in V\left(\mathcal{T}^{\prime}\right)$ are either comparable or upper neighbours of the same vertex $\gamma \in \mathcal{T}^{\prime}$, then $v(\alpha) \neq v(\beta)$.

By virtue of (6.2), $v$ can be interpreted as mapping increasing paths $\pi \subset \mathcal{T}$ to 'real' paths $P \subset G$. For example, if $\pi=\xi_{0} \ldots \xi_{n}$ is a $\phi-\alpha$ path in $\mathcal{T}$, then $v(\pi)$ is the path $v\left(\xi_{0}\right) \ldots v\left(\xi_{n}\right)$ in $G$, which is none other than the path $\alpha \subset G$ 
itself. More generally, if $\pi \subset \mathcal{T}$ is an increasing path from $\alpha$ to $\beta$, and if $v(\alpha)=: a$, then $v(\pi)$ is precisely the subpath $a \beta$ of the path $\beta \subset G$. When $\mathcal{T}^{\prime}$ is any subgraph of $\mathcal{T}$ other than a path, we may use $v\left(\mathcal{T}^{\prime}\right)$ informally to denote the set $\left\{v(\alpha) \mid \alpha \in \mathcal{T}^{\prime}\right\} \subset V(G)$.

Let $\mathcal{T}^{\prime}$ be a good family tree. We shall call $\alpha \in V\left(\mathcal{T}^{\prime}\right)$ a burst vertex of $\mathcal{T}^{\prime}$ if $\alpha$ has infinite degree. (Thus, the burst vertices of $\mathcal{T}^{\prime}$ are precisely its branch vertices other than $\phi$ when $\mathcal{T}^{\prime}$ is viewed as a long-rooted $\mathrm{T} T_{\omega}$.) When $\alpha \in \mathcal{T}^{\prime}$ is a burst vertex, the burst at $\alpha$ in $\mathcal{T}^{\prime}$ is the union of all the paths $\alpha \ldots \gamma \subset \mathcal{T}^{\prime}$ such that $\gamma$ is minimal among the burst vertices above $\alpha$. These paths will be called the segments of the burst; the burst itself will be denoted by $B_{\mathcal{T}^{\prime}}(\alpha)$. When $\mathcal{T}^{\prime}=\mathcal{T}$ or the precise choice of the good family tree considered is irrelevant, we may abbreviate $B_{\mathcal{T}^{\prime}}(\alpha)$ to $B(\alpha)$. Thus, a burst $B(\alpha)$ is a subdivided infinite star, contained in $\mathcal{T}$ and centred at $\alpha$, its segments being the star's subdivided edges.

Let us call a burst $B(\alpha)$ blocked by a vertex $b \in G$ if infinitely many of its segments contain a vertex $\beta \neq \alpha$ with $v(\beta)=b$. If $B(\alpha)$ is not blocked by any vertex of $G$, it will be called free.

Passing to a good family tree $\mathcal{T}^{\prime} \subset \mathcal{T}$ if necessary, we may assume that the following is true for $\mathcal{T}$ :

(6.3) Whenever a burst $B(\alpha)$ is blocked, there is a vertex $b_{\alpha} \in G$ such that every segment $\pi$ of $B(\alpha)$ contains a (unique) vertex $\beta_{\pi} \neq \alpha$ with $v\left(\beta_{\pi}\right)=b_{\alpha}$.

Moreover, since any segment of a burst $B_{\mathcal{T}^{\prime}}(\alpha)$ in a good family tree $\mathcal{T}^{\prime}$ contains a segment of $B_{\mathcal{T}}(\alpha)$, we see that (6.3) is in fact true for all good family trees. (Recall that any good family tree is a subtree of $\mathcal{T}$.)

Let $\alpha_{0}$ be the lowest burst vertex in $\mathcal{T}$. Then $\left\lfloor\alpha_{0}\right\rfloor$ is the long root of $\mathcal{T}$, and $\mathcal{T} \geqslant \alpha_{0} \simeq \mathrm{T} T_{\omega}$. Applying Lemma 4.1 to $\mathcal{T} \geqslant \alpha_{0}$, we see that either there is a burst vertex $\alpha \in \mathcal{T}$ such that all bursts at vertices in $\mathcal{T} \geq \alpha=: \widetilde{\mathcal{T}}$ are free, or $\mathcal{T} \geqslant \alpha_{0}$ has a subtree $\widetilde{\mathcal{T}} \simeq \mathrm{T} T_{\omega}$ (whose minimal vertex is a branch vertex) such that for every branch vertex $\alpha$ of $\widetilde{\mathcal{T}}$ the burst $B_{\mathcal{T}}(\alpha)$ is blocked. In either case, let $\mathcal{T}^{\prime}$ be obtained from $\widetilde{\mathcal{T}}$ by adding the $\phi-\widetilde{\mathcal{T}}$ path in $\mathcal{T}$ as a long root. Then $\mathcal{T}^{\prime}$ is a good family tree. Moreover, in the first case every burst in $\mathcal{T}^{\prime}$ is free, while in the second case (6.3) implies that every burst in $\mathcal{T}^{\prime}$ is blocked. Hence, passing to this good family tree $\mathcal{T}^{\prime}$, we may assume that either all bursts in $\mathcal{T}$ are blocked or all bursts in $\mathcal{T}$ are free.

Let us quickly dispose of the case when all bursts are free, by showing how to find a $\mathrm{T} T_{\omega}$ in $G$. If all the bursts in $\mathcal{T}$ are free then, for every finite set $F \subset V(G)$ and every burst vertex $\alpha$, there is a segment of $B(\alpha)$ such that for every vertex $\beta \neq \alpha$ on this segment we have $v(\beta) \notin F$. Using (6.2), and following the method of the proof of Lemma 4.2, we may thus construct a good family tree $\mathcal{T}^{\prime} \subset \mathcal{T}$ on which the function $v$ is injective. (Choose segments $\pi=\alpha \ldots \gamma$ inductively as in the standard construction of $T^{*} \simeq T_{\omega}$, in such 
a way that $v(\propto \pi)$ is disjoint from $v\left(\pi^{\prime}\right)$ for the - finitely many-segments $\pi^{\prime}$ chosen earlier.) The image of $\mathcal{T}^{\prime}$ under $v$ then spans a long-rooted $\mathrm{TT}_{\omega}$ in $G$.

We may therefore assume from now on that all bursts in $\mathcal{T}$ are blocked. By (6.3), this assumption carries over to all good family trees:

(6.4) If $\mathcal{T}^{\prime}$ is a good family tree and $\alpha$ is a burst vertex of $\mathcal{T}^{\prime}$, then $B_{\mathcal{T}^{\prime}}(\alpha)$ is blocked by the vertex $b_{\alpha} \in G$.

Let us continue our investigation of the bursts in good family trees. We claim the following:

(6.5) If $\alpha$ is a burst vertex in a good family tree $\mathcal{T}^{\prime}$, then $v(\alpha) \in T^{\prime}$.

Indeed, suppose that $v(\alpha) \notin T^{\prime}$. Let $\beta$ be the highest vertex of $\mathcal{T}^{\prime}$ below $\alpha$ such that $v(\beta) \in T^{\prime} ; \beta$ exists, because $v(\phi) \in T^{\prime}$. Let $\pi_{0}, \pi_{1}, \ldots$ be the segments of $B_{\mathcal{T}}(\alpha)$, and for each $n \in \mathbb{N}$ let $\rho_{n}$ be a ray in $\mathcal{T}^{\prime}$ which starts at $\beta$ and contains $\pi_{n}$. Since these rays are increasing in $\mathcal{T}^{\prime}$, they correspond to rays $v\left(\rho_{n}\right)=: R_{n}$ in $G$ (recall the remark following (6.2)). Each of the rays $R_{n}$ starts at $v(\beta)$; let $t_{n}=v\left(\tau_{n}\right)$ be its first inner vertex on $T^{\prime}$. This vertex $t_{n}$ exists by Lemma 3.9, and it comes after $v(\alpha)$ on $R_{n}$ by the choice of $\beta$. Moreover, the vertices $t_{n}$ are all in $T^{\prime}[v(\alpha)]$, which is finite by Lemma 3.4. Therefore infinitely many of the $t_{n}$ are the same ( $t$, say), and the corresponding paths $P_{n}:=R_{n} t$ form an infinite family of $T^{\prime}-T^{\prime}$ paths in $G$ from $v(\beta)$ to $t$. The paths $P_{n}$ are distinct, because $v$ is injective on the set of upper neighbours of $\alpha$ in $\mathcal{T}^{\prime}$. Therefore $(v(\beta), t)$ is a bad pair. Since $P_{n}$ is a subpath of the path $\tau_{n} \in V\left(\mathcal{T}^{\prime}\right)$ (for any $n$ ), this contradicts the fact that $V\left(\mathcal{T}^{\prime}\right)$ is a good family of paths in $G$. This completes the proof of (6.5).

Let $\alpha \in \mathcal{T}$ be a burst vertex. Let us show that $v\left(\stackrel{\alpha}{\alpha} \dot{\beta}_{\pi}\right) \cap T^{\prime} \neq \emptyset$ for all but finitely many exceptional segments $\pi$ of $B(\alpha)$. In other words: for almost every segment of $B(\alpha)$ the corresponding path in $G$ meets $T^{\prime}$ before it hits the blocking vertex $b_{\alpha}$.

Suppose, for a contradiction, that $B(\alpha)$ has infinitely many segments $\pi_{0}, \pi_{1}, \ldots$ such that $v\left(\stackrel{\circ}{\alpha} \pi_{n}{\stackrel{\circ}{\beta_{n}}}_{\pi_{n}}\right) \cap T^{\prime}=\emptyset$ for every $n$. We argue as in the proof of (6.5). For each $n$, let $P_{n}:=v\left(\pi_{n}\right)$, and let $t_{n}$ be the first vertex on $b_{\alpha} P_{n}$ that is in $T^{\prime}$; such a vertex exists, because the last vertex of $P_{n}$ corresponds to another burst vertex of $\mathcal{T}$, and is therefore in $T^{\prime}$ by (6.5). Since each of the vertices $t_{n}$ is in $T^{\prime}\left[b_{\alpha}\right]$, which is finite, infinitely many of them are the same: $t$, say. The corresponding paths $P_{n} t$ are therefore $T^{\prime}-T^{\prime}$ paths in $G$ from $v(\alpha)$ to $t$; they are distinct, because the upper neighbours of $\alpha$ in $\mathcal{T}$ have distinct images under $v$. Hence $(v(\alpha), t)$ is a bad pair, a contradiction.

For every burst vertex $\alpha$, let us remove from $\mathcal{T}$ the finitely many exceptional segments of $B(\alpha)$ (and all the vertices above these segments). So, by passing to a good family tree $\mathcal{T}^{\prime} \subset \mathcal{T}$ if necessary, we may assume that, for every burst vertex $\alpha \in \mathcal{T}$ and every segment $\pi$ of $B(\alpha)$, the corresponding path 
$v(\pi) \subset G$ meets $T^{\prime}$ before it hits the blocking vertex $b_{\alpha}$. Again, this carries over to all good family trees:

(6.6) If $\alpha$ is a burst vertex in a good family tree $\mathcal{T}^{\prime}$, and if $\pi$ is a segment of $B_{\mathcal{T}^{\prime}}(\alpha)$, then $v\left(\stackrel{\alpha}{\alpha} \dot{\beta}_{\pi}\right) \cap T^{\prime} \neq \emptyset$. The first vertex $\tau$ of $\dot{\alpha} \pi \dot{\beta}_{\pi}$ with $v(\tau) \in T^{\prime}$ will be denoted by $\tau_{\pi}$, and we write $t_{\pi}$ for $v\left(\tau_{\pi}\right)$.

When $R$ is a normal ray, let us extend the definition of $R$-height - and the notion of '(.)-clear' associated with it - from $V(G)$ to $V(\mathcal{T})$ in the natural way, setting ht $(\alpha ; R):=\operatorname{ht}(v(\alpha) ; R)$.

Let $\alpha$ be a burst vertex in some good family tree. We shall call its burst $B(\alpha)$ in this tree a long burst if there exists a normal ray $R \subset G$ such that the neighbourhood of $\alpha$ in $B(\alpha)$ has unbounded $R$-height. Otherwise, $B(\alpha)$ is a short burst.

For each burst vertex $\alpha \in \mathcal{T}$ where $B(\alpha)$ is a long burst, let us pick a normal ray $R_{\alpha} \subset G$ such that the neighbourhood of $\alpha$ in $B(\alpha)$ has unbounded $R_{\alpha}$-height. We may then select infinitely many segments of $B(\alpha)$ so that the neighbours of $\alpha$ on these segments have distinct $R_{\alpha}$-heights. The $R_{\alpha}$-height on the set of these neighbours then tends to infinity. Passing to a good family tree $\mathcal{T}^{\prime} \subset \mathcal{T}$ if necessary, we may therefore assume that, for each burst vertex $\alpha \in \mathcal{T}$ with $B(\alpha)$ long, the $R_{\alpha}$-height of its neighbours in $B(\alpha)$ tends to infinity.

The same is true then for every infinite subset of the set of upper neighbours of $\alpha$ in $\mathcal{T}$. Our assumption therefore extends to all good family trees:

(6.7) Whenever $B(\alpha)$ is a long burst in some good family tree, the $R_{\alpha}$-height of the neighbours of $\alpha$ in $B(\alpha)$ tends to infinity.

As earlier when we considered free versus blocked bursts, let us apply Lemma 4.1 to the tree $\mathcal{T} \geqslant \alpha_{0} \simeq \mathrm{T} T_{\omega}$, where $\alpha_{0}$ is the lowest burst vertex in $\mathcal{T}$. The lemma now implies that either there is a burst vertex $\alpha \in \mathcal{T}$ such that all bursts at vertices in $\mathcal{T} \geq \alpha=: \widetilde{\mathcal{T}}$ are short, or else $\mathcal{T} \geqslant \alpha_{0}$ has a subtree $\widetilde{\mathcal{T}} \simeq \mathrm{T} T_{\omega}$ (whose minimal vertex is a branch vertex) such that for every branch vertex $\alpha$ of $\widetilde{\mathcal{T}}$ the burst $B_{\mathcal{T}}(\alpha)$ is long. Let $\mathcal{T}^{\prime}$ be obtained from $\widetilde{\mathcal{T}}$ by adding the $\phi-\widetilde{\mathcal{T}}$ path in $\mathcal{T}$ as a long root. Then $\mathcal{T}^{\prime}$ is a good family tree. Moreover, in the first case every burst in $\mathcal{T}^{\prime}$ is short, while in the second case (6.7) (applied to $\mathcal{T}$ ) implies that every burst in $\mathcal{T}^{\prime}$ is long. Passing to this good family tree $\mathcal{T}^{\prime}$, we may thus assume that either all bursts of $\mathcal{T}$ are short or all bursts of $\mathcal{T}$ are long.

We shall consider these two cases separately.

Case 1: All bursts in $\mathcal{T}$ are short.

We shall construct a bundle graph in $G$ by induction, adding one bundle at a time. The bundle graph will consist of the image of a ray in $\mathcal{T}$, together with bundles made up from some of the bursts along the ray. 
To be precise, let us construct a sequence $G_{0} \subset G_{1} \subset \ldots$ of subgraphs of $G$, and a sequence $\alpha_{0}<\alpha_{1}<\ldots$ of vertices of $\mathcal{T}$, so that the following four conditions are satisfied for every $n \in \mathbb{N}$ :

(i) $G_{n}$ is a partial bundle graph (with $n$ bundles) on the path $\alpha_{n} \subset G$;

(ii) $\alpha_{n}$ is a burst vertex of $\mathcal{T}$;

(iii) the vertex $v\left(\alpha_{n}\right)$ has degree 1 in $G_{n}$;

(iv) the image of $V\left(\mathcal{T}^{>\alpha_{n}}\right)$ under the map $v$ is disjoint from $V\left(G_{n}\right)$.

Roughly speaking, condition (i) asserts that our construction has been successful so far, while conditions (ii)-(iv) will enable us to continue the construction without running into obstructions.

Note that the induction starts, with $\alpha_{0}$ as any burst vertex of $\mathcal{T}$ and $G_{0}$ as the path $\alpha_{0}$ in $G$. Note also that once we have constructed the graphs $G_{n}$, their union is a bundle graph in $G$, as required.

Let us then turn to the induction step. Suppose we have constructed $G_{0} \subset \ldots \subset G_{n}$ and $\alpha_{0}<\ldots<\alpha_{n}$ in accordance with conditions (i)-(iv). Put $\alpha:=\alpha_{n}$ and $a:=v(\alpha)$, and let $\Pi$ be an infinite set of segments of $B(\alpha)$. Recall that, for each $\pi \in \Pi, t_{\pi}=v\left(\tau_{\pi}\right)$ is the first vertex of $v\left(\stackrel{\alpha}{\alpha} \dot{\circ}_{\pi}\right)$ in $T^{\prime}$.

Note that the vertices $t_{\pi}$ cannot be the same (say $t$ ) for infinitely many $\pi \in \Pi$ : the pair $(a, t)$ would then be bad, contradicting the fact that $a v(\pi) t$ is a $T^{\prime}-T^{\prime}$ path contained in the path $\tau_{\pi} \in V(\mathcal{T})$ (for any $\pi$ with $t_{\pi}=t$ ). Moreover, by Lemma 3.3 each $t_{\pi}$ is comparable with $a$. As $G$ has only finitely many vertices below $a$, it follows that $a<t_{\pi}$ for all but finitely many $\pi \in \Pi$. Replacing $\Pi$ with a suitable infinite subset if necessary, we may therefore assume the following:

(6.8) The vertices $t_{\pi}(\pi \in \Pi)$ are all distinct and lie strictly above $a$ in $T^{\prime}$.

Let

$$
H:=\bigcup_{\pi \in \Pi} a T^{\prime} t_{\pi}
$$

$H$ is an infinite subtree of $T^{\prime}$. Hence, by König's theorem, $H$ contains either a ray or a vertex of infinite degree. If $H$ contains a ray, then a tail of this ray is increasing in $T$, and is therefore a tail of some normal ray $R$. By construction of $H$ and Lemma 3.5, the vertices $t_{\pi}$ have unbounded $R$-height. The neighbours of $\alpha$ in $B(\alpha)$ then also have unbounded $R$-height (again by Lemma 3.5), which contradicts the fact that $B(\alpha)$ is short. Hence $H$ has a vertex $z$ of infinite degree.

Replacing $\Pi$ with a suitable infinite subset, we may assume that every $t_{\pi}$ lies above $z$, and that $\left\lfloor t_{\pi}\right\rfloor \cap\left\lfloor t_{\pi^{\prime}}\right\rfloor=\lfloor z\rfloor$ whenever $\pi \neq \pi^{\prime}$ (Fig. 7).

By Lemma 3.2, this implies that

$$
\text { If } \pi, \pi^{\prime} \in \Pi \text { are distinct, then any } t_{\pi}-t_{\pi^{\prime}} \text { path in } G \text { meets }\lfloor z\rfloor \text {. }
$$


FiguRE 7. The graph $H \subset T^{\prime}$

For each $\pi \in \Pi$, let

$$
P_{\pi}:=v\left(\pi \beta_{\pi}\right)
$$

recall that $v\left(\beta_{\pi}\right)$ is the blocking vertex $b_{\alpha}$ for $B(\alpha)$. Then

$$
\mathcal{P}_{\Pi}:=\left\{P_{\pi} \mid \pi \in \Pi\right\}
$$

is an infinite set of $a-b_{\alpha}$ paths in $G$ whose second vertices are distinct. By Lemma 2.3, the union $\bigcup \mathcal{P}_{\Pi}$ of these paths contains a bundle or a fan; we claim it must contain a bundle:

(6.10) $\bigcup \mathcal{P}_{\Pi}$ contains a bundle $B$ as specified in Lemma 2.3 (i).

To prove $(6.10)$, suppose $\bigcup \mathcal{P}_{\Pi}$ contains no such bundle. Then, by Lemma 2.3, $\bigcup \mathcal{P}_{\Pi}$ contains a fan $F$ as specified in Lemma 2.3 (ii). We shall construct, inductively, an infinite sequence $Q_{0}, Q_{1}, \ldots$ of disjoint paths in $F$, each joining two vertices of $\left\{t_{\pi} \mid \pi \in \Pi\right\}$. (Fig. 8). This will contradict (6.9), which asserts that these vertices are pairwise separated in $G$ by the finite subgraph $\lfloor z\rfloor$.

FIGURE 8. Finding the paths $Q_{0}, Q_{1}, \ldots$ in $F$ 
Let $k \in \mathbb{N}$ and assume that $Q_{i}$ has been chosen, for every $i<k$, so that $a \notin Q_{i}$. Let $\ell \in \mathbb{N}$ be large enough that none of the paths $Q_{i}$ meets $v_{\ell} R$ or any of the spokes $S_{n}$ with $n>\ell$. By Lemma 3.9, $v_{\ell} R$ has a vertex $t$ on $T^{\prime}$. Let $m$ be such that $t \in v_{m-1} R v_{m}$. By the choice of $F$ (see Lemma 2.3), the path $S_{m} v_{m} R v_{m-1}$ is an initial segment of a path $P_{\pi} \in \mathcal{P}_{\Pi}$. Since $t_{\pi}$ is the first vertex of $\stackrel{\circ}{a} P_{\pi}$ in $T^{\prime}$, it precedes $t$ on $P_{\pi}$ or coincides with it, and is therefore itself on the path $S_{m} v_{m} R v_{m-1}$. Now pick $t^{\prime} \in \stackrel{v}{v}_{m} R \cap T^{\prime}$, say between $v_{n-1}$ and $v_{n}$ on $R$, find $\pi^{\prime} \in \Pi$ such that $S_{n} v_{n} R v_{n-1}$ is an initial segment of $P_{\pi^{\prime}}$, and note that $t_{\pi^{\prime}} \in S_{n} v_{n} R v_{n-1}$. Let $Q_{k}$ be the $t_{\pi}-t_{\pi^{\prime}}$ path in $S_{m} \cup v_{m-1} R v_{n} \cup S_{n}$. Then $a \notin Q_{k}$, and $Q_{k} \cap Q_{i}=\emptyset$ for all $i<k$. This completes the induction step, and hence the proof of (6.10).

Note that, according to its specification by Lemma 2.3 (i), the bundle $B$ in (6.10) is an $a-x$ bundle on a path $P \in \mathcal{P}_{\Pi}$, where $x$ is some vertex of $P$. Moreover, each of the $a-x$ paths in $B$ is an initial segment of some path $P_{\pi} \in \mathcal{P}_{\Pi}$; let $F_{\pi} \subset P_{\pi}$ be the corresponding fibre of $B$.

Our aim is to obtain our new partial bundle graph $G_{n+1}$ by adding the bundle $B$ on to $G_{n}$. To ensure that $G_{n+1}$ will be compatible with the disjointness condition (iv), however (for some suitable $\alpha_{n+1} \in \mathcal{T}$ ), we first have to delete some fibres from $B$.

We first wish to delete those fibres $F_{\pi}$ from $B$ which do not contain $t_{\pi}$. Before we do this, we have to check that only finitely many fibres of $B$ will be affected, i.e. that $t_{\pi} \in F_{\pi}$ for all but finitely many of the fibres $F_{\pi}$. If this is not the case, then $F_{\pi} \subset G \backslash T^{\prime}$ for infinitely many $\pi \in \Pi$ (by definition of the vertices $t_{\pi}$; recall that $F_{\pi}$ is an initial segment of $\left.\stackrel{\circ}{a} P_{\pi}\right)$. Then $t_{\pi} \in T^{\prime}[x]$ for all these $\pi$, and hence $t_{\pi} \in\lfloor x\rfloor$ by Lemma 3.4. Since $\lfloor x\rfloor$ is finite, this means that the vertices $t_{\pi}$ coincide for infinitely many of the fibres $F_{\pi}$, contradicting (6.8).

So, removing finitely many fibres from $B$ if necessary, we may assume that $t_{\pi} \in F_{\pi}$ for each fibre $F_{\pi}$ of $B$. Again removing finitely many fibres, we may assume that no fibre of $B$ meets $\lfloor z\rfloor$. Hence, (6.9) implies the following:

(6.11) The fibres of $B$ are pairwise separated by $\lfloor z\rfloor$ in $G$.

(In particular, $x \in\lfloor z\rfloor$.)

We are now almost ready to attach the bundle $B$ to our partial bundle graph $G_{n}$. Choose $\beta \in B(\alpha)$ so that $v(\beta)=x$ and the path $\alpha \mathcal{T} \beta$ has length at least 2. Among all $\gamma \geqslant \beta$, choose one with $|\gamma \cap\lfloor z\rfloor|$ maximal, and pick a burst vertex $\alpha_{n+1}>\gamma$. By the choice of $\gamma$, we have

$$
v\left(\mathcal{T}^{\geq \alpha_{n+1}}\right) \cap\lfloor z\rfloor=\emptyset .
$$

Indeed, if $\delta \in \mathcal{T}^{\geq \alpha_{n+1}}$ (and thus $\delta>\gamma$ ), then the path $\delta \subset G$ is a proper extension of the path $\gamma$. The choice of $\gamma$ implies that $\delta \cap\lfloor z\rfloor \subset \gamma$; in particular, $v(\delta)$ (which is the last vertex of $\delta$ ) is not in $\lfloor z\rfloor$. 
As $G\left[v\left(\mathcal{T}^{\geq \alpha_{n+1}}\right)\right]$ is connected, (6.11) and (6.12) imply that at most one fibre of $B$ contains a vertex in $v\left(\mathcal{T}^{\geq \alpha_{n+1}}\right)$; delete any such fibre from $B$. Moreover, only finitely many fibres of $B$ can have a vertex on the (finite) path $\alpha_{n+1} \backslash \alpha_{n}\left(=v\left(\stackrel{\circ}{\alpha}_{n} \mathcal{T} \alpha_{n+1}\right)\right)$; delete those fibres as well. We may now set

$$
G_{n+1}:=G_{n} \cup \alpha_{n+1} \cup B
$$

Let us quickly verify the induction hypothesis for $n+1$. We certainly have $G_{n} \subset G_{n+1}$, and $\alpha_{n+1}$ is a burst vertex of $\mathcal{T}$ (assertion (ii)) strictly above $\alpha_{n}$.

For assertion (i), recall first that $\alpha_{n+1}$ is an extension of the path $\alpha_{n}$, and that $\alpha_{n+1} \backslash \alpha_{n} \cap G_{n}=\emptyset$ by condition (iv) for $n$, because $V\left(\alpha_{n+1} \backslash \alpha_{n}\right) \subset$ $v\left(\mathcal{T}^{>\alpha_{n}}\right)$. Hence, $G_{n}$ is a partial bundle graph on $\alpha_{n+1}$. The interior of the new bundle $B$ is contained in the union of the paths $v(\stackrel{\circ}{\pi})$, where $\pi$ ranges over the segments of the burst $B(\alpha)=B\left(\alpha_{n}\right)$. The interior of $B$ is therefore again disjoint from $G_{n}$ by condition (iv) for $n$. As the interior of $B$ also avoids the path $\alpha_{n+1} \backslash \alpha_{n}$ (by construction; recall the deletion of fibres just before the definition of $\left.G_{n+1}\right), B$ is also a bundle on the path $\alpha_{n+1}$. Finally, if $B^{\prime}$ is any of the bundles of $G_{n}$, then both vertices of $B^{\prime} \cap \alpha_{n+1}$ precede $v\left(\alpha_{n}\right)(=a)$ on $\alpha_{n+1}$ by condition (iii) for $n$, and hence they also precede the two vertices of $B \cap \alpha_{n+1}$. Hence $G_{n+1}$ is a partial bundle graph, with $n+1$ bundles, on the path $\alpha_{n+1} \subset G$.

By the choice of $\alpha_{n+1}$ as strictly above $\beta$, we know that $v\left(\alpha_{n+1}\right)$ has degree 1 in $G_{n+1}$ (assertion (iii)).

For assertion (iv), note that $v\left(\mathcal{T}>\alpha_{n+1}\right)$ is disjoint from $V\left(G_{n}\right)$ by condition (iv) for $n$, since $\alpha_{n+1}>\alpha_{n}$ and hence $\mathcal{T}^{>\alpha_{n+1}} \subset \mathcal{T}^{>\alpha_{n}}$. It is certainly disjoint from $V\left(\alpha_{n+1}\right)=v\left(\phi \mathcal{T} \alpha_{n+1}\right)$, and it is finally disjoint from the interior of $B$, since we deleted the one fibre of $B$ that could possibly meet $v\left(\mathcal{T} \geq \alpha_{n+1}\right)$. Hence, $v\left(\mathcal{T}^{>\alpha_{n+1}}\right)$ is disjoint from $V\left(G_{n+1}\right)$.

This completes the induction step in our construction of a bundle graph in $G$, and so completes Case 1 .

Case 2: All bursts of $\mathcal{T}$ are long.

Recall that, by (6.7), each burst vertex $\alpha \in \mathcal{T}$ is associated with a normal ray $R_{\alpha}$, so that the $R_{\alpha}$-height of the neighbours of $\alpha$ in $B(\alpha)$ tends to infinity. Let again $\alpha_{0}$ be the lowest burst vertex in $\mathcal{T}$. Applying Lemma 4.2 to $\mathcal{T} \geqslant \alpha_{0}$, we see that $\mathcal{T}$ contains a good family tree $\mathcal{T}^{\prime}$ with the property that the rays $R_{\alpha}$ are either all the same or all distinct for the burst vertices $\alpha \in \mathcal{T}^{\prime}$. Let us rename $\mathcal{T}^{\prime}$ as $\mathcal{T}$, and address the two cases in turn.

If all the rays $R_{\alpha}$ are the same, say $R$, choose a sequence of burst vertices $\alpha_{0}<\alpha_{1}<\ldots$ in $\mathcal{T}$. Then $v\left(\alpha_{0}\right), v\left(\alpha_{1}\right), \ldots$ are distinct vertices of $G$, whose neighbourhoods have unbounded $R$-height. By Lemma 3.11 (ii), this implies that $G$ contains a $\mathrm{T} K_{\omega}$, and hence a $\mathrm{T} T_{\omega}$.

We may therefore assume from now on that the rays $R_{\alpha}$ are all distinct: 
(6.12) If $\alpha$ and $\alpha^{\prime}$ are distinct burst vertices of $\mathcal{T}$, then $R_{\alpha}$ and $R_{\alpha^{\prime}}$ are distinct normal rays in $G$.

Similarly to our construction of a bundle graph in the short burst case, we shall now construct a fan graph in $G$ along the image of a ray from $\mathcal{T}$. The construction will be more complicated, however: the individual fans will be made not just from bursts $B(\alpha)$ along that ray, but partly also from the rays $R_{\alpha}$ corresponding to these bursts.

To be precise, let us construct a sequence $G_{0} \subset G_{1} \subset \ldots$ of subgraphs of $G$, and a sequence $\beta_{0}<\beta_{1}<\ldots$ of vertices of $\mathcal{T}$, so that the following three conditions are satisfied for every $n \in \mathbb{N}$ :

(i) $G_{n}$ is a partial fan graph (with $n$ fans) on the path $\beta_{n} \subset G$;

(ii) for every burst vertex $\gamma>\beta_{n}$ of $\mathcal{T}$, the $R_{\gamma}$-height of $G_{n}$ is bounded;

(iii) there is a burst vertex $\nu_{n}>\beta_{n}$ such that the image of $V\left(\stackrel{\circ}{\beta}_{n} \mathcal{T} \nu_{n} \cup \mathcal{T}^{>\nu_{n}}\right)$ under the map $v$ is disjoint from $V\left(G_{n}\right)$.

Again, roughly speaking, condition (i) asserts that our construction has been successful so far, while conditions (ii) and (iii) will enable us to continue the construction.

We apply induction on $n$. Taking $G_{0}:=\beta_{0}:=\phi$, and letting $\nu_{0}$ be any burst vertex of $\mathcal{T}$, we see that the induction starts. Note also that once we have constructed the graphs $G_{n}$ for all $n \in \mathbb{N}$, their union is a fan graph in $G$, as desired.

Let us then turn to the induction step. Suppose we have constructed subgraphs $G_{0} \subset \ldots \subset G_{n}$ of $G$ and found vertices $\beta_{0}<\ldots<\beta_{n}$ and $\nu_{n}$ of $\mathcal{T}$ in accordance with conditions (i)-(iii). In order to define $G_{n+1}$, we wish to construct a new fan to add to $G_{n}$. However, it turns out to be difficult to pinpoint directly a fan that misses the whole of $G_{n}$, and which can be added to $G_{n}$ in a manner that allows the induction to continue. We shall instead define two fans (to be called $F$ and $F^{\prime}$ ), and our aim will be to show that at least one of these fans is suitable for attachment to $G_{n}$.

Put $\alpha:=\nu_{n}$ and $R:=R_{\alpha}$, and let $a:=v(\alpha)$. As the path $\alpha \subset G$ is finite, condition (ii) implies that the $R$-height of $G_{n} \cup \alpha$ is bounded. We may therefore choose a neighbour $\delta=\delta_{0}$ of $\alpha$ in $B(\alpha)$ that is $\left(G_{n} \cup \alpha\right)$-clear with respect to $R$ (cf. (6.7)). Let $r_{0} \in R$ be the $R$-height of $\delta$. Let us further choose segments $\pi_{1}, \pi_{2}, \ldots$ of $B(\alpha)$ such that, writing $\delta_{i}$ for the neighbour of $\alpha$ on $\pi_{i}$ and $r_{i}$ for the $R$-height of $\delta_{i}$, we have $r_{0}<r_{1}<r_{2}<\ldots$ For each $i \in \mathbb{N}$, denote $v\left(\delta_{i}\right)$ by $d_{i}$, and let $P_{i}$ be a $d_{i}-R$ path in $G$ that ends in $r_{i}$ (using the definition of $R$-height). See Figure 9 .

Since $P_{i} \stackrel{\circ}{r}_{i} \cap R=\emptyset$, Lemma 3.5 implies that the $R$-height is constant on $P_{i} \stackrel{\circ}{r}_{i}$, and thus equal to the $R$-height of $d_{i}$. Hence we have:

(6.13) For each $i \in \mathbb{N}$, every vertex of $P_{i}$ has $R$-height $r_{i}$. 
Figure 9. Constructing a fan from $B(\alpha)$ and $R_{\alpha}$

Define a fan $F$ from $a$ to $d:=d_{0}$, as follows. The spine of $F$ is the ray $P_{0} r_{0} R$. Its spokes are the paths $a d_{i} P_{i}$ for $i>0$. (In other words, the $i$ th spoke consists of the edge $a d_{i}$ followed by the path $P_{i}$.) By (6.13), the spokes of $F$ are pairwise disjoint (except for $a$ ) and avoid $P_{0}$, so $F$ is indeed a fan. As further consequences of (6.13), we have the following:

(6.14) The interior of $F$ is $\left(G_{n} \cup \alpha\right)$-clear with respect to $R$.

(6.15) The $R$-height of $F$ tends to infinity.

In order to define our second fan $F^{\prime}$, choose a burst vertex $\alpha^{\prime}>\delta$, let $R^{\prime}:=R_{\alpha^{\prime}}$, and put $a^{\prime}:=v\left(\alpha^{\prime}\right)$. By condition (ii), the $R^{\prime}$-height of $G_{n} \cup \alpha^{\prime}$ is bounded. Moreover, since $R^{\prime} \neq R$, Lemma 3.8 and (6.15) tell us that the $R^{\prime}$-height of $F$ is bounded. Choose a neighbour $\delta^{\prime}=\delta_{0}^{\prime}$ of $\alpha^{\prime}$ in $B\left(\alpha^{\prime}\right)$ that is $\left(G_{n} \cup F \cup \alpha^{\prime}\right)$-clear with respect to $R^{\prime}$. Put $d^{\prime}:=v\left(\delta^{\prime}\right)$, and define $\pi_{i}^{\prime}, \delta_{i}^{\prime}, r_{i}^{\prime}, d_{i}^{\prime}$, $P_{i}^{\prime}$ and a fan $F^{\prime}$ from $a^{\prime}$ to $d^{\prime}$ exactly as above (Fig. 10).

Statements 6.13-6.15 then have the following equivalents for $F^{\prime}$ :

$\left(6.13^{\prime}\right)$ For each $i \in \mathbb{N}$, every vertex of $P_{i}^{\prime}$ has $R^{\prime}$-height $r_{i}^{\prime}$.

$\left(6.14^{\prime}\right)$ The interior of $F^{\prime}$ is $\left(G_{n} \cup F \cup \alpha^{\prime}\right)$-clear with respect to $R^{\prime}$.

$\left(6.15^{\prime}\right)$ The $R^{\prime}$-height of $F^{\prime}$ tends to infinity.

Setting $z:=\max \left(R \cap R^{\prime}\right)$, we see by (6.15) and Lemma 3.7 that there are vertices in $F$ with $R^{\prime}$-height $z$. The $R^{\prime}$-height of $\delta^{\prime}$, and hence of every vertex in $F^{\prime}$, is therefore greater than $z$ :

(6.16) $F^{\prime}$ is $\lfloor z\rfloor$-clear with respect to $R^{\prime}$. 
FIgURE 10. The fans $F$ and $F^{\prime}$

Now let $U:=\{x \in F \mid$ ht $(x ; R) \leqslant z\}$, and put $W:=U \cup\lfloor z\rfloor$. Note that $U$, and hence $W$, is finite by (6.15). We claim that

(6.17) $W$ separates $F$ from $F^{\prime}$ in $G$.

Indeed, consider $F^{\prime}$ and $F \backslash W$. Each vertex of $F^{\prime}$ has $R^{\prime}$-height greater than $z$, while each vertex of $F \backslash W$ has $R$-height greater than $z$. By Lemma 3.6, this implies that $\lfloor z\rfloor$ separates $F^{\prime}$ from $F \backslash W$ in $G$, so $W$ separates $F$ from $F^{\prime}$ as claimed.

Among all $\gamma \geqslant \delta^{\prime}$, choose one with $|V(\gamma) \cap W|$ maximal, and pick a burst vertex $\nu>\gamma$. By the choice of $\gamma$, we have $v\left(\mathcal{T}^{\geq \nu}\right) \cap W=\emptyset$. Since $G\left[v\left(\mathcal{T}^{\geq \nu}\right)\right]$ is connected, this implies that $v\left(\mathcal{T}^{\geq \nu}\right)$ meets at most one of $F$ and $F^{\prime}$. Let $F^{\prime \prime} \in\left\{F, F^{\prime}\right\}$ be such that $v(\mathcal{T} \geq \nu) \cap V\left(F^{\prime \prime}\right)=\emptyset$, and define $\alpha^{\prime \prime}, R^{\prime \prime}, a^{\prime \prime}, \delta^{\prime \prime}$, $d^{\prime \prime}, r_{i}^{\prime \prime}$ and $P_{i}^{\prime \prime}$ accordingly. Thus,

(6.18) $F^{\prime \prime}$ is a fan from $a^{\prime \prime}$ to $d^{\prime \prime}$ which avoids $v\left(\mathcal{T}^{\geq \nu}\right)$.

Moreover, statements $6.13-6.15$ and their analogues for $F^{\prime}$ imply the following:

$\left(6.13^{\prime \prime}\right)$ For each $i \in \mathbb{N}$, every vertex of $P_{i}^{\prime \prime}$ has $R^{\prime \prime}$-height $r_{i}^{\prime \prime}$.

$\left(6.14^{\prime \prime}\right)$ The interior of $F^{\prime \prime}$ is $\left(G_{n} \cup \alpha^{\prime \prime}\right)$-clear with respect to $R^{\prime \prime}$.

$\left(6.15^{\prime \prime}\right)$ The $R^{\prime \prime}$-height of $F^{\prime \prime}$ tends to infinity. 
Our final task now is to attach this new fan to $G_{n}$. Set

$$
X:=\left\{v(\beta) \mid \delta^{\prime \prime} \leqslant \beta \leqslant \nu\right\} \cap V\left(F^{\prime \prime}\right) .
$$

Note that $X \neq \emptyset$, as $d^{\prime \prime} \in X$. Let $Y \subset X$ consist of the vertices in $X$ of maximal $R^{\prime \prime}$-height. Pick a vertex $b \in Y$ as follows. If $Y$ contains a vertex on $R^{\prime \prime}$, let $b$ be this (unique) vertex. If $Y \cap V\left(R^{\prime \prime}\right)=\emptyset$, then all the vertices of $Y$ lie on a common spoke of $F^{\prime \prime}$ or on $P_{0}^{\prime \prime}$ (by $\left(6.13^{\prime \prime}\right)$ ); let $b$ be the vertex of $Y$ closest to $R^{\prime \prime}$ along this spoke (respectively, along $P_{0}^{\prime \prime}$ ).

Define a fan $E$ from $a^{\prime \prime}$ to $b$ as follows. Let $r$ be the $R^{\prime \prime}$-height of $b$. As the spokes of $E$, take those spokes of $F^{\prime \prime}$ whose last vertex is above $r$. If $b \in R^{\prime \prime}$ (and $r=b$ ), take $b R^{\prime \prime}$ as the spine of $E$. If $b \notin R^{\prime \prime}$, say $b \in P_{k}^{\prime \prime}$, let the spine of $E$ be the ray $b P_{k}^{\prime \prime} r R^{\prime \prime}$. In either case, $E$ is a fan from $a^{\prime \prime}$ to $b$ contained in $F^{\prime \prime}$ (Fig 11).

Figure 11. The fan $E \subset F^{\prime \prime}$

Set $\nu_{n+1}:=\nu$, and let $\beta_{n+1}$ be the unique vertex $\beta \leqslant \nu$ with $v(\beta)=b$. Note that, in fact, $\delta^{\prime \prime} \leqslant \beta<\nu$ by (6.18) and the definition of $X$, so

$$
\alpha^{\prime \prime}<\delta^{\prime \prime} \leqslant \beta_{n+1}<\nu_{n+1} .
$$

Finally, let

$$
G_{n+1}:=G_{n} \cup \beta_{n+1} \cup E .
$$

Let us verify the induction hypothesis for $n+1$. We certainly have $G_{n} \subset$ $G_{n+1}$ and $\beta_{n}<\beta_{n+1}$.

For assertion (i), note first that the path $\beta_{n+1} \backslash \beta_{n}=v\left(\stackrel{\circ}{n}_{n} \mathcal{T} \beta_{n+1}\right)$ avoids $G_{n}$ by condition (iii) for $n$. The interior of $E$ avoids $G_{n} \cup \alpha^{\prime \prime}$ by $\left(6.14^{\prime \prime}\right)$ (since $E \subset F^{\prime \prime}$ ), and it avoids the path $\nu_{n+1} \backslash \alpha^{\prime \prime}$ by the choice of $b$. (Recall that $\delta^{\prime \prime}$ is 
an upper neighbour of $\alpha^{\prime \prime}$ in $\mathcal{T}$.) In particular, $E$ is a fan on $\beta_{n+1} \backslash \beta_{n}$ (recall that $\beta_{n}<\alpha \leqslant \alpha^{\prime \prime}$ ), and (i) follows.

For a proof of assertion (ii), let $\gamma$ be any burst vertex above $\beta_{n+1}$. Then $\gamma \neq \alpha^{\prime \prime}$, so the $R_{\gamma}$-height of $E$ is bounded by $\left(6.15^{\prime \prime}\right)$ and Lemma 3.8. As the path $\beta_{n+1}$ is finite, this implies that (ii) carries over from $n$ to $n+1$.

To verify (iii), note that $G_{n}$ avoids the set

$$
V:=v\left(\stackrel{\circ}{\beta}_{n+1} \mathcal{T} \nu_{n+1} \cup \mathcal{T}^{>\nu_{n+1}}\right)
$$

by condition (iii) for $n$, because $\nu_{n+1}>\nu_{n}$. We saw in the proof of (i) that the interior of $E$ avoids the path $\nu_{n+1} \backslash \alpha^{\prime \prime}$, so $E$ avoids $V$ by (6.18). The path $\beta_{n+1}$ avoids $V$ by (6.2). Hence $G_{n+1}$ avoids $V$.

This completes the induction step in our construction of a fan graph in $G$, and so completes Case 2. The proof of Theorem 6.1 is thus complete.

\section{The bounded graph theorem}

To conclude the paper, let us restate our principal result and complete its formal proof. Recall that $\mathfrak{b}$ denotes the least cardinal such that there exists a family of $\mathfrak{b}$ distinct $\mathbb{N} \rightarrow \mathbb{N}$ sequences which are not dominated by a common $\mathbb{N} \rightarrow \mathbb{N}$ sequence. (So, $\omega<\mathfrak{b} \leqslant 2^{\omega}$, and $\omega_{1}=\mathfrak{b}=2^{\omega}$ with CH.) Recall further that $I_{\mathfrak{b}}$ denotes the disjoint union of $\mathfrak{b}$ rays, and that $T_{\omega}$ is the tree of countably infinite regular degree. As in the Introduction, let $B$ and $F$ be the prototype bundle graph and the prototype fan graph, respectively (see Figs. 1 and 2).

Theorem 7.1. (Bounded graph theorem)

A graph is bounded if and only if it contains none of the following graphs as a topological subgraph: $I_{\mathfrak{b}} ; T_{\omega} ; B ; F$.

Proof. Since boundedness is closed under taking subgraphs, the 'only if' part follows from the fact that the four forbidden graphs and their subdivisions are themselves unbounded.

For the 'if' part, let us suppose that a graph $G$ contains no subdivision of any of $I_{\mathfrak{b}}, T_{\omega}, B$ or $F$; we show that $G$ is bounded. Since $G \nsupseteq I_{\mathfrak{b}}$, fewer than $\mathfrak{b}$ of its components contain a ray. By definition of $\mathfrak{b}$, we may therefore assume that $G$ is connected. Since $G$ does not contain a $\mathrm{T} K_{\omega}$ (which would contain a $\mathrm{T} T_{\omega}$ ), $G$ has a normal spanning tree by Halin's theorem (3.1). By Theorem 6.1 , then, there is no good family of paths in $G$, so $G$ is bounded by Theorem 5.1.

Remark. If the Continuum Hypothesis is assumed then $\omega_{1}=\mathfrak{b}=2^{\omega}$, so Theorem 7.1 implies the bounded graph conjecture as stated in the Introduction. 
In the remainder of this section we list a few immediate consequences of the bounded graph theorem. The following three corollaries were already discussed in the Introduction.

Corollary 7.2. Subdivisions of bounded graphs are bounded, and subdivisions of unbounded graphs are unbounded.

Corollary 7.3. Let $G$ be a graph. Unless $G$ contains $\mathfrak{b}$ disjoint rays (in which case it is trivially unbounded), $G$ is bounded if and only if all its countable subgraphs are bounded.

Corollary 7.4. If a countable graph admits some unbounded labelling then all its injective labellings are unbounded.

Our next corollary is a strengthening of Theorem 5.1. Let us call a nonempty family $\mathcal{P}$ of finite paths propagating if it satisfies condition (i) of the definition of a good family, i.e., if for every path $P \in \mathcal{P}$ there exists an $n \in \mathbb{N}$ such that $P$ has infinitely many extensions in $\mathcal{P}$ of length $n$. Clearly, any graph with a propagating family of paths must be unbounded, and any graph containing a subdivision of $T_{\omega}, B$ or $F$ contains a propagating family of paths.

Corollary 7.5. A graph $G$ not containing $I_{\mathfrak{b}}$ is bounded if and only if there is no propagating family of paths in $G$.

One may ask whether or not boundedness can also be characterized by the exclusion of minors rather than topological subgraphs. Since the minor relation is transitive, this would require that boundedness be closed under taking minors, i.e. that minors of bounded graphs should again be bounded. With the most general definition of an infinite minor, as given in $\S 2$, this is easily seen to be false: the infinite grid, for example, is locally finite and hence bounded, but contracting every second vertical ray yields a graph which contains both a copy of $F$ and a bundle graph (and is therefore unbounded).

However, a slightly less general definition of an infinite minor (which still generalizes the usual finite definition) will work: if we require that all the branch sets be finite - i.e., if all our minors are obtained by first taking a subgraph and then contracting finite connected parts of that subgraph - then boundedness does turn out to be closed under taking minors. Indeed, if $H$ is a bounded graph and $H \supseteq H^{\prime}=\mathrm{H} G$ with all branch sets finite, it is not difficult to see that any $\mathrm{T} B, \mathrm{~T} F, \mathrm{~T} T_{\omega}$ or $I_{\mathfrak{b}}$ subgraph of $G$ could be lifted to a corresponding $\mathrm{T} B$, $\mathrm{T} F$, $\mathrm{T} T_{\omega}$ or $I_{\mathfrak{b}}$ subgraph of $H^{\prime}$, and hence of $H$, contradicting the boundedness of $H$. Therefore $G$, too, must be bounded:

Corollary 7.6. Boundedness is closed under taking minors with finite branch sets. 
By Lemma 2.1, we thus have the following forbidden minor version of Theorem 7.1:

Theorem 7.7. A graph is bounded if and only if it contains none of the following graphs as a minor (with all branch sets finite): $I_{\mathfrak{b}} ; T_{\omega} ; B ; F$.

\section{References}

[1] R. Diestel, Graph Decompositions - a study in infinite graph theory, Oxford University Press, Oxford 1990.

[2] R. Diestel, On spanning trees and $k$-connectedness in infinite graphs, J. Combin. Theory $B$ (to appear).

[3] R. Halin, Simplicial decompositions of infinite graphs, in: (B. Bollobás, Ed.) Advances in Graph Theory (Annals of Discrete Mathematics 3), North-Holland Publ. Co., Amsterdam/London 1978.

[4] R. Halin, Some problems and results in infinite graphs, in: (L.D. Andersen et al., Eds.) Graph Theory in Memory of G. A. Dirac (Annals of Discrete Mathematics 41), NorthHolland Publ. Co., Amsterdam/London 1989.

[5] R. Halin, Bounded graphs, in: (R. Diestel, Ed.) Directions in infinite graph theory and combinatorics (Annals of Discrete Mathematics), to appear.

[6] H. A. Jung, Wurzelbäume und unendliche Wege in Graphen, Math. Nachr. 41 (1969), $1-22$.

[7] D. König, Theorie der endlichen und unendlichen Graphen, Akademische Verlagsgesellschaft, Leipzig 1936 (reprinted: Chelsea, New York 1950).

[8] H. J. Prömel and B. Voigt, Aspects of Ramsey Theory, Springer Verlag, in preparation.

[9] R. Rado, Universal graphs and universal functions, Acta Arith. 9 (1964), 331-340.

Reinhard Diestel

Faculty of Mathematics (SFB 343)

Bielefeld University

D-4800 Bielefeld

Germany
Imre Leader

Department of Pure Mathematics

16 Mill Lane

Cambridge CB2 1SB

England 